\title{
Lipogenesis inhibitors: therapeutic opportunities and challenges
}

\section{Battsetseg Batchuluun ${ }^{1}$, Stephen L. Pinkosky ${ }^{2}$ and Gregory R. Steinberg ${ }^{1 凶}$}

Abstract | Fatty acids are essential for survival, acting as bioenergetic substrates, structural components and signalling molecules. Given their vital role, cells have evolved mechanisms to generate fatty acids from alternative carbon sources, through a process known as de novo lipogenesis (DNL). Despite the importance of DNL, aberrant upregulation is associated with a wide variety of pathologies. Inhibiting core enzymes of DNL, including citrate/isocitrate carrier (CIC), ATP-citrate lyase (ACLY), acetyl-CoA carboxylase (ACC) and fatty acid synthase (FAS), represents an attractive therapeutic strategy. Despite challenges related to efficacy, selectivity and safety, several new classes of synthetic DNL inhibitors have entered clinical-stage development and may become the foundation for a new class of therapeutics.

\section{NAFLD}

Nonalcoholic fattly liver disease (NAFLD) includes a spectrum of metabolic diseases ranging from simple steatosis to cirrhosis in the liver.
${ }^{1}$ Centre for Metabolism, Obesity and Diabetes Research, Department of Medicine and Department of Biochemistry and Biomedical Sciences, McMaster University, Hamilton, Ontario, Canada.

${ }^{2}$ Esperion Therapeutics, Ann Arbor, MI, USA.

凶e-mail: gsteinberg@ mcmaster.ca

https://doi.org/10.1038/ s41573-021-00367-2
Fatty acids are essential for cell survival as they serve as key structural components of cell membranes and important signalling molecules. Fatty acids are also the most calorically dense form of energy storage with the conversion of excess glucose into fatty acids protecting against glucotoxicity and providing a much larger energy reserve than glycogen for times of nutrient scarcity. Given the vital roles of fatty acids, cells have evolved mechanisms to maintain them at adequate levels. This includes mechanisms to take up exogenous fatty acids but also to generate fatty acids from alternative carbon sources through a series of enzymatic reactions, a process highly conserved across phyla known as de novo lipogenesis $(\mathrm{DNL})^{1}$.

DNL is initiated when excess substrate availability, relative to cellular energy demands, leads to increases in mitochondrial citrate, which is exported from mitochondria into the cytosol by the mitochondrial citrate/ isocitrate carrier (CIC; also known as CTP and SLC25A1) (FIG. 1). This cytosolic citrate is then converted into fatty acids by a series of biosynthetic reactions catalysed by ATP-citrate lyase (ACLY), acetyl-CoA carboxylase (ACC; also known as ACACA) and fatty acid synthase (FAS; also known as FASN). The expression of these enzymes differs across tissues and stages of development (for example, proliferation or quiescence). Expression and activity are also acutely and chronically regulated through transcriptional control and post-translational modifications that are linked to nutritional status (for example, fasting and feeding) and substrate availability (for example, fatty acids suppress DNL) (BOX 1).

Although DNL is vital to maintain whole-body and cellular homeostasis, chronic elevations are associated with the development of a broad spectrum of diseases and disorders including cardiovascular disease (CVD) ${ }^{2,3}$, nonalcoholic fatty liver disease (NAFLD) $)^{4,5}$, type 2 diabetes $(\mathrm{T} 2 \mathrm{D})^{5,6}$, numerous cancers ${ }^{7,8}$, viral infections ${ }^{9,10}$, autoimmune diseases $^{11,12}$, acne vulgaris ${ }^{13}$, neurodegeneration $^{14}$ and ageing ${ }^{15}$. This suggests that pharmacological inhibition may be beneficial across multiple disease areas (BOX 2; Supplementary Fig. 1). Several natural products have been identified as inhibitors of DNL and these have been adopted as a cornerstone for the development of synthetic inhibitors that display improved bioavailability, efficacy and specificity. Recently, some of these compounds have reached clinical stage development and have been approved for the treatment of hyperlipidaemia ${ }^{16}$ or are in late-stage development for NAFLD and oncology.

However, there are still many important questions that remain to be answered as it is currently unclear whether systemic or organ-specific inhibition of DNL should be targeted and what degree of inhibition is necessary to avoid potential side effects such as defects in fetal development ${ }^{17}$, platelet production ${ }^{18}$ or muscle dysfunction ${ }^{19}$. It is also unclear under what conditions inhibition of the classical DNL pathway may be bypassed by the scavenging of alternative carbon sources, through acetyl-CoA synthetase $e^{20,21}$, ketoacid dehydrogenase ${ }^{22}$ or isocitrate dehydrogenases ${ }^{23-25}$, and therefore whether combination therapies or changes in diet may be required. Although there are several important targets that indirectly influence lipogenesis (for example, fructokinase, glucokinase or the glucagon receptor, sterol regulatory element-binding protein 1 (SREBP1) and liver X receptor (LXR) $)^{26,27}$ or are involved in downstream lipid processing (for example, SCD1, DGAT1 and DGAT2 ${ }^{28,29}$, here, we specifically review recent advances in the development of inhibitors that directly target core components within the lipogenic pathway, 
namely, citrate/isocitrate carrier (CIC), ATP-citrate lyase (ACLY), ACC and fatty acid synthase (FAS).

\section{Physiological and pathological roles of DNL enzymes}

The physiological regulation of DNL is complex, differing widely between cell types and with nutritional status. As discussed in this Review, studies from mice genetically lacking Slc25a1, Acly, Acaca and Fas have confirmed the importance of DNL in lipogenic tissues such as liver and adipose tissue, but also revealed unexpected biological consequences in cell types thought to have limited capacity for DNL. These findings indicate a much broader role for DNL in regulating the production of lipids under a wider array of physiological functions than initially appreciated (FIG. 2). Although a detailed description of the complex physiology that regulates lipogenesis (reviewed in REFS ${ }^{30,31}$ ) is not within the scope of this Review, an overview of the key physiological and pathological roles of DNL enzymes is provided below. Importantly, the information obtained from genetic studies provides crucial insight into both the opportunities and challenges of developing DNL inhibitors.

\section{Energy intake and expenditure}

Activity of the DNL pathway appears to regulate energy intake and expenditure. For example, mice that lack Fas have reduced food intake ${ }^{32}$, whereas food intake is

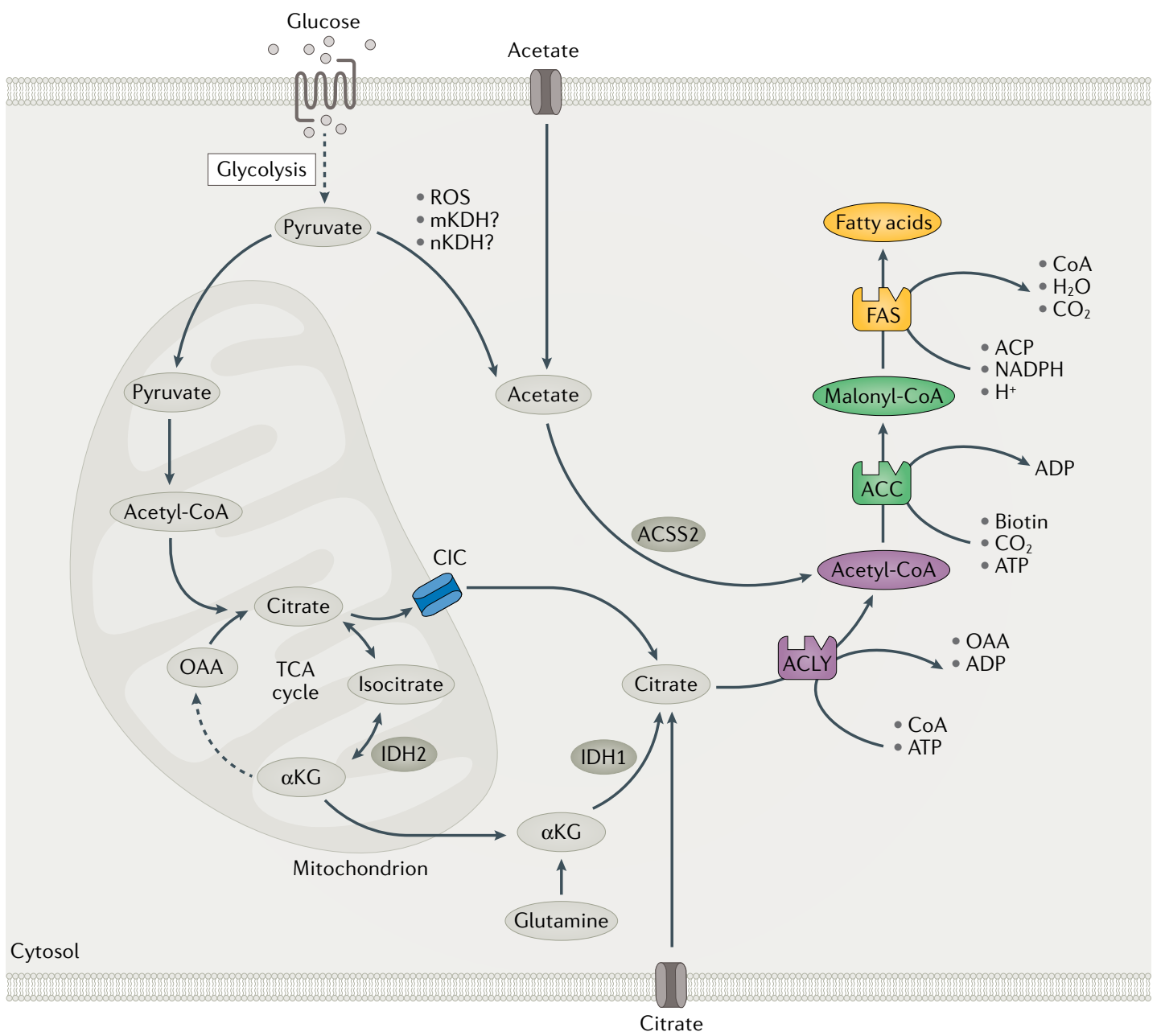

Fig. 1 | Overview of DNL. A series of coordinated enzymatic reactions takes place during fatty acid biosynthesis. Typically, pyruvate produced by glycolysis is converted in the mitochondrion into acetyl-CoA, which enters the tricarboxylic acid (TCA) cycle to produce citrate. In conditions of carbohydrate excess, citrate is exported to the cytosol by the citrate/isocitrate carrier (CIC) and is broken down to acetyl-CoA and oxaloacetate (OAA) by ATP-citrate lyase (ACLY). Acetyl-CoA is subsequently carboxylated by acetyl-CoA carboxylase (ACC) to generate malonyl-CoA, which is considered the first committed metabolic intermediate in fatty acid synthesis. Utilizing seven malonyl-CoA molecules and one acetyl-CoA primer, the synthesis of palmitate (16:0 fatty acid) is completed by repeating a cycle of condensation, reduction, condensation and dehydration catalysed by fatty acid synthase (FAS). An alternative carbon source of de novo lipogenesis (DNL) is acetate, which can be produced de novo from glucose through non-enzymatic and enzymatic reactions. Acetyl-CoA synthetase 2 (ACSS2) catalyses the reaction of acetate and CoA to form acetyl-CoA, which is subsequently used for fatty acid biosynthesis. With hypoxia or CIC deficiency another alternative pathway for DNL is reductive carboxylation of glutamine via cytosolic isocitrate dehydrogenase 1 (IDH1) and mitochondrial IDH2. aKG, a-ketoglutarate; ACP, acyl carrier protein; mKDH, mitochondrial ketoacid dehydrogenase; $\mathrm{nKDH}$, nuclear ketoacid dehydrogenase; ROS, reactive oxygen species. 


\section{Box 1 | Transcriptional control of DNL enzymes}

De novo lipogenesis (DNL) is fundamental for the survival of multicellular organisms. However, it is also an energy-intensive process that requires 7 ATP and 14 NADPH molecules to convert acetyl-CoA into palmitate. As such, numerous overlapping biological pathways have been developed to tightly match pathway flux with nutrient availability.

Transcriptional regulation of the citrate/isocitrate carrier (CIC), ATP-citrate lyase (ACLY), acetyl-CoA carboxylase (ACC) and fatty acid synthase (FAS) is governed by a set of three transcription factors called sterol regulatory element-binding proteins (SREBPs), carbohydrate-responsive element-binding proteins (ChREBPs) and liver $X$ receptors (LXRs) ${ }^{29,281}$ (FIG. 3). Although cholesterol and fatty acids are both synthesized from acetyl-CoA, their biosynthetic pathways are largely regulated by distinct SREBPs with DNL being dependent on SREBP1a and SREBP1c whereas cholesterol synthesis is primarily regulated by SREBP2 (REF. ${ }^{282}$ ). In the liver, the expression of CIC, ACLY, ACC and FAS are increased by SREBP1c in response to glucose and insulin, while being repressed by fatty acids ${ }^{283}$. The expression of DNL genes is also altered by ChREBP, which exists as two isoforms, ChREBP $\alpha$ and ChREBP $\beta$, that have differential tissue expression profiles.

Upon ingestion of carbohydrates, ChREBPa is activated causing nuclear import and binding to carbohydrate-responsive elements in promoters of lipogenic genes, promoting transcription; a response that is further amplified by ChREBP $\beta$, which is constitutively active ${ }^{281}$. LXRs have a central role in the transcriptional control of SREBP1c by insulin ${ }^{283}$, and although ChREBP was originally suggested to be a target gene of $\mathrm{LXRs}^{284}$, this has not been observed in all studies ${ }^{285}$. The activity of all three transcription factors is altered by a multitude of adaptor proteins, ubiquitin ligases, transcriptional activators and repressors as well as epigenetic and post-translational modifications $^{29,281}$.

\section{Futile cycling}

A process of using energy

in two opposing metabolic pathways without a net effect.

NASH

Nonalcoholic steatohepatitis (NASH) is an advanced form of nonalcoholic fatty liver disease that is characterized by liver steatosis, inflammation and fibrosis.

Obesity

A condition characterized

by the excessive accumulation of fat in the body.

Insulin resistance

An impaired glucose-lowering effect of insulin increased in mice that lack $A c c^{33}$. Mechanistically, the divergent effects of FAS and ACC on food intake can potentially be explained through differential effects on malonyl-CoA, which has been shown to suppress food intake: FAS inhibition increases malonyl-CoA, whereas ACC inhibition reduces it. Consistent with changes in malonyl-CoA, stereotactic delivery of malonyl-CoA decarboxylase (MCD) into the hypothalamus increases food intake ${ }^{34}$, whereas food intake is reduced in mice with constitutively active ACC1 and ACC2 isoforms ${ }^{35}$. These data suggest that inhibition of FAS suppresses food intake whereas inhibition of ACC stimulates food intake.

In both mice and humans, brown adipose tissue (BAT) is switched on in response to increases in nutrients (diet-induced thermogenesis) or cold (adaptive thermogenesis $)^{36,37}$. The activation of BAT in rodents and humans increases glucose uptake; however, recent studies have found that surprisingly, the primary function of this glucose is not to support glycolysis but instead to fuel $\mathrm{DNL}^{38}$. Consistent with this concept, both cold exposure and high carbohydrate availability increase DNL within BAT $^{39}$, suggesting that BAT primarily utilizes endogenous triglycerides to fuel thermogenesis. Paradoxically, reductions in adipose tissue Fas leads to reductions in body mass ${ }^{40}$ due to enhanced local sympathetic nervous system activity, which causes the browning of the white fat ${ }^{41}$. These data suggest that counterintuitively inhibiting DNL in adipose tissue may increase whole-body energy expenditure. Future studies are required to selectively inhibit ACLY, ACC and FAS within BAT and white adipose tissue (WAT) to directly quantify the importance of the DNL pathway for regulating futile cycling and energy expenditure.

\section{Lipid deposition: NAFLD-NASH}

Individuals with nonalcoholic fatty liver disease (NAFLD) have increased rates of DNL and this is a major factor contributing to increased lipid deposition ${ }^{4,5}$. Consistent with these observations, the expression of CIC, ACLY, ACC and FAS are increased in the liver of patients with NAFLD or nonalcoholic steatohepatitis $(\mathrm{NASH})^{42}$. In mice fed a high-fat diet (HFD), liver-specific deletion of the Slc25a1 gene reduces liver steatosis ${ }^{43}$. In $o b / o b$ mice fed a high-carbohydrate diet, transient genetic inhibition of ACLY using small interfering RNA (siRNA) reduces liver lipid content ${ }^{44}$. Similarly, when Acly is selectively removed from hepatocytes of adult mice with NASH induced by a high-fat and high-fructose diet, there are reductions in liver steatosis (G.R.S., unpublished observations). By contrast, lifelong inhibition of liver Acly in mice fed high levels of fructose does not lower liver fat, potentially because of upregulation of acetate production by the gut microbiome and compensatory upregulation of acetate-CoA synthetase 2 (ACSS2) $\left(\mathrm{REF}^{45}\right)$. However, it should be noted that the high levels of fructose used in this study did not promote obesity, NAFLD or $\mathrm{NASH}^{45}$; thus, the therapeutic relevance of the findings is currently unclear.

Genetic inhibition of liver $A c c 1$ (REF. ${ }^{46}$ ) or both $A c c 1$ and $A c c 2\left(\mathrm{REFS}^{47,48}\right)$ lowers liver fat in mice fed a highcarbohydrate diet independently of changes in adiposity. Conversely, mice with constitutively active ACC1 and ACC2 isoforms, owing to lack of AMP-activated protein kinase (AMPK) inhibitory phosphorylation, develop greater steatosis and fibrosis than controls when fed a high-carbohydrate diet ${ }^{49}$. These data indicate that inhibition of ACC can exert positive effects on lowering steatosis and fibrosis. However, an important consequence of inhibiting liver ACC is the development of hypertriglyceridaemia owing to reductions in liver polyunsaturated fatty acids, which increase SREBP1c and the expression of glycerol-3-phosphate acyltransferase (GPAT), the rate-limiting enzyme for triglyceride synthesis ${ }^{48}$. Although most studies have indicated positive effects of ACC inhibition on liver steatosis, in one study genetic inhibition led to increases in liver lipids attributed to hyperacetylation of mitochondrial proteins, potentially due to accumulation of acetyl-CoA and lower fatty acid oxidation $^{50}$. The phenotype of these liver-specific ACC null mice was similar to that of mice lacking FAS, which also have increased steatosis owing to reductions in fatty acid oxidation ${ }^{51}$. These studies in ACC-null and FAS-null mice highlight complex interactions that may limit therapeutic application in NAFLD and NASH.

\section{Insulin sensitivity: type 2 diabetes}

Insulin resistance is a hallmark of type 2 diabetes (T2D). Rates of DNL in the liver are inversely correlated with hepatic and whole-body insulin sensitivity in humans ${ }^{5,52}$. Liver-specific deletion of Slc25a1 improves glucose tolerance in mice fed a control carbohydrate diet or HFD ${ }^{43}$. siRNA-mediated suppression of ACLY expression reduces fasting glucose and improves glucose tolerance in $o b / o b$ mice fed a high-carbohydrate diet ${ }^{44}$. Similar observations are made when ACLY is selectively deleted from hepatocytes of mice fed a high-fat and high-fructose 
diet for 16 weeks (G.R.S., unpublished observations). Genetic inhibition of ACC2 in the muscle ${ }^{53}$ or ACC1 and ACC2 in the liver ${ }^{47}$ leads to improvements in muscle and liver insulin sensitivity, respectively, whereas mice with constitutively active ACC1 and ACC2 isoforms have worse insulin resistance ${ }^{49,54}$. These data indicate that inhibition of DNL and/or upregulation of fatty acid oxidation within muscle and liver of mice can improve insulin sensitivity.

In addition to muscle and liver, adipose tissue is also crucial for regulation of insulin sensitivity ${ }^{55,56}$. In rodents, WAT has rates of DNL comparable to those of liver during the postprandial period ${ }^{57}$. However, with obesity, rates of adipose tissue DNL decline in both rodents $^{58}$ and humans $s^{59}$, despite only modest reductions in insulin-simulated glucose uptake ${ }^{60}$. Recent studies indicate that this reduction in adipose tissue DNL occurs rapidly and continues to decline with worsening obesity and insulin resistance and is associated with reductions in the expression of ACLY, ACC, FAS ${ }^{61}$ and carbohydrate-responsive element-binding protein- $\beta$ $(\text { ChREBP } \beta)^{62}$, suggesting that inhibition of adipose tissue DNL may be detrimental to whole-body insulin sensitivity. Consistent with this concept, when ACLY fat-specific null mice are challenged with a high-sucrose diet, female mice develop a lipodystrophy-like phenotype that is associated with hepatic lipid accumulation and insulin resistance ${ }^{63}$. Similar observations are also observed in ACC1 fat-specific null mice ${ }^{64}$. These data

\section{Box 2 | Emerging applications of lipogenesis inhibitors}

In addition to the widely studied therapeutic application of inhibitors of de novo liopogenesis (DNL) enzymes in metabolic diseases and cancer, emerging evidence indicates that lipogenesis inhibitors may also be beneficial in several other disorders.

During viral infections, lipogenesis is upregulated to meet the high demand for membrane synthesis required for viral replication. As such, blocking lipogenesis has emerged as a potential antiviral therapeutic option. For instance, lipogenesis inhibitors have been demonstrated to block the infection of hepatitis $\mathrm{C}$ virus ${ }^{286}, \mathrm{HIV}^{287}$, West Nile virus $^{288}$, rotavirus $^{289}$, Epstein-Barr virus ${ }^{290}$, dengue virus ${ }^{291}$, Japanese encephalitis virus ${ }^{291}$ and chikungunya virus ${ }^{291}$. Lipogenesis inhibitors could also potentially be beneficial against the COVID-19 pandemic, as like all viruses, SARS-CoV-2 relies on newly synthesized phospholipids for its replication ${ }^{292}$. Consistent with this concept, orlistat and TVB-2640 inhibit the replication of SARS-CoV-2 variants ${ }^{293}$.

Another emerging area for lipogenesis inhibitors involves the treatment of acne vulgaris. Excess sebum production is an important cause of acne vulgaris, and studies using isotope labelling have revealed that $~ 80 \%$ of the sebum lipid in humans is derived from $\mathrm{DNL}^{13}$. In early-stage clinical trials, topical applications of acetyl-CoA carboxylase (ACC) inhibitors olumacostat glasaretil ${ }^{294}$ and PF-05175157 (REF. ${ }^{13}$ ) showed beneficial effects for treating acne vulgaris; however, it appears that neither agent has proceeded further with clinical development.

Multiple studies of early inhibitors soraphen A and TOFA suggest a potential benefit of ACC inhibition on several other pathological conditions. Soraphen $A$ attenuates Thelper $17\left(T_{H} 17\right)$ cell-mediated autoimmune disease ${ }^{98}$, inhibiting effector $T$ cell expansion in graft-versus-host disease ${ }^{295}$, and improves neurological outcomes after ischaemic stroke by preserving the regulatory $T$ cell and $\mathrm{T}_{\mathrm{H}} 17$ cell balance ${ }^{296}$. More recently, it was demonstrated that soraphen $A$ blocks autophagy in ageing yeast ${ }^{297}$ and attenuates undirected endothelial cell migration, which is a process implicated in many severe diseases ${ }^{298}$. Recent studies suggest that TOFA is involved in survival of memory T cells during chronic infections ${ }^{299}$ and in reduction of pro-inflammatory signalling in cystic fibrosis ${ }^{300}$. Although these findings are intriguing, owing to the potential off-target effects of these compounds, conclusions about the therapeutic benefit of ACC inhibition derived from these studies should be interpreted with caution until they are replicated using more-specific inhibitors. indicate an important role for adipose tissue DNL in maintaining whole-body insulin sensitivity, potentially by protecting the liver from developing steatosis. Additional studies characterizing transient knock-down of ACLY and ACC in fully differentiated adipocytes of obese adult mice, rather than lifelong deletion ${ }^{20,63,64}$, could better inform the potential protective role of adipose tissue $\mathrm{DNL}$ in insulin resistance.

The inability of pancreatic $\beta$-cells to secrete sufficient amounts of insulin to maintain euglycaemia is a hallmark of T2D. Available evidence suggests that ACC may be important for pancreatic $\beta$-cell function, as deletion of ACC impairs glucose-stimulated insulin secretion ex vivo and insulin tolerance in vivo ${ }^{65}$. Mechanistically, ACC is abundant in $\beta$-cells, whereas FAS is expressed at low levels, leading to a rise in malonyl-CoA levels following glucose stimulation ${ }^{66}$. As a result, fatty acid oxidation is inhibited by elevated malonyl-CoA, increasing the availability of cytosolic long-chain fatty acyl-CoA (LCFA-CoA) for lipid signalling to cellular processes involved in insulin secretion ${ }^{67}$. In addition, recent studies have found that ACC is important for promoting $\beta$-cell mass in mice ${ }^{65}$. These data suggest that inhibition of ACC in pancreatic islets may lead to impaired insulin secretion and reduced $\beta$-cell mass that could contribute to T2D.

\section{Cardiovascular disease}

Elevations in liver DNL lead to increases in plasma VLDL and LDL, major risk factors of cardiovascular disease (CVD) mortality ${ }^{68,69}$. Recent studies have found that incident heart failure and CVD mortality ${ }^{2,70}$ are positively associated with increases in fatty acids derived from DNL. Consistent with this concept, genetic variants in $A C L Y$ are associated with reduced plasma LDL and decreased cardiovascular events ${ }^{3}$. Similarly, polymorphisms of $A C L Y$ and $A C C$ are associated with lower triglycerides following dietary fish oil supplementation ${ }^{71}$. Surprisingly, to the best of our knowledge, no studies have examined the effects of genetic inhibition of liver Acly, Acc or Fas on established models of atherosclerotic development. However, given the effects of ACC inhibition in promoting hypertriglyceridaemia ${ }^{48}$, it might be anticipated to enhance atherosclerosis. By contrast, a deficiency in liver ACLY lowers triglycerides in mice (G.R.S., unpublished observations). The reasons for the opposing effects of ACLY and ACC inhibition on circulating triglycerides is unclear; however, it could be linked to the concomitant suppression of cholesterol synthesis unique to ACLY inhibition and/or the reciprocal effect that ACLY and ACC inhibition has on levels of acetyl-CoA, which may be important to support protein acetylation. These data indicate that while ACC inhibition may be effective at reducing liver steatosis, it has detrimental effects on CVD risk profile that would need to be managed with other lipid-lowering therapies such as fish oil ${ }^{71}$, DGAT inhibition ${ }^{72}$ or PPARa agonists such as fenofibrate ${ }^{73}$.

Macrophages are also crucial for the development of atherosclerotic CVD as they take up and store lipid, triggering inflammation within the atherosclerotic lesion. The activity of $\mathrm{ACLY}^{74}$ and $\mathrm{FAS}^{75}$ is increased in 


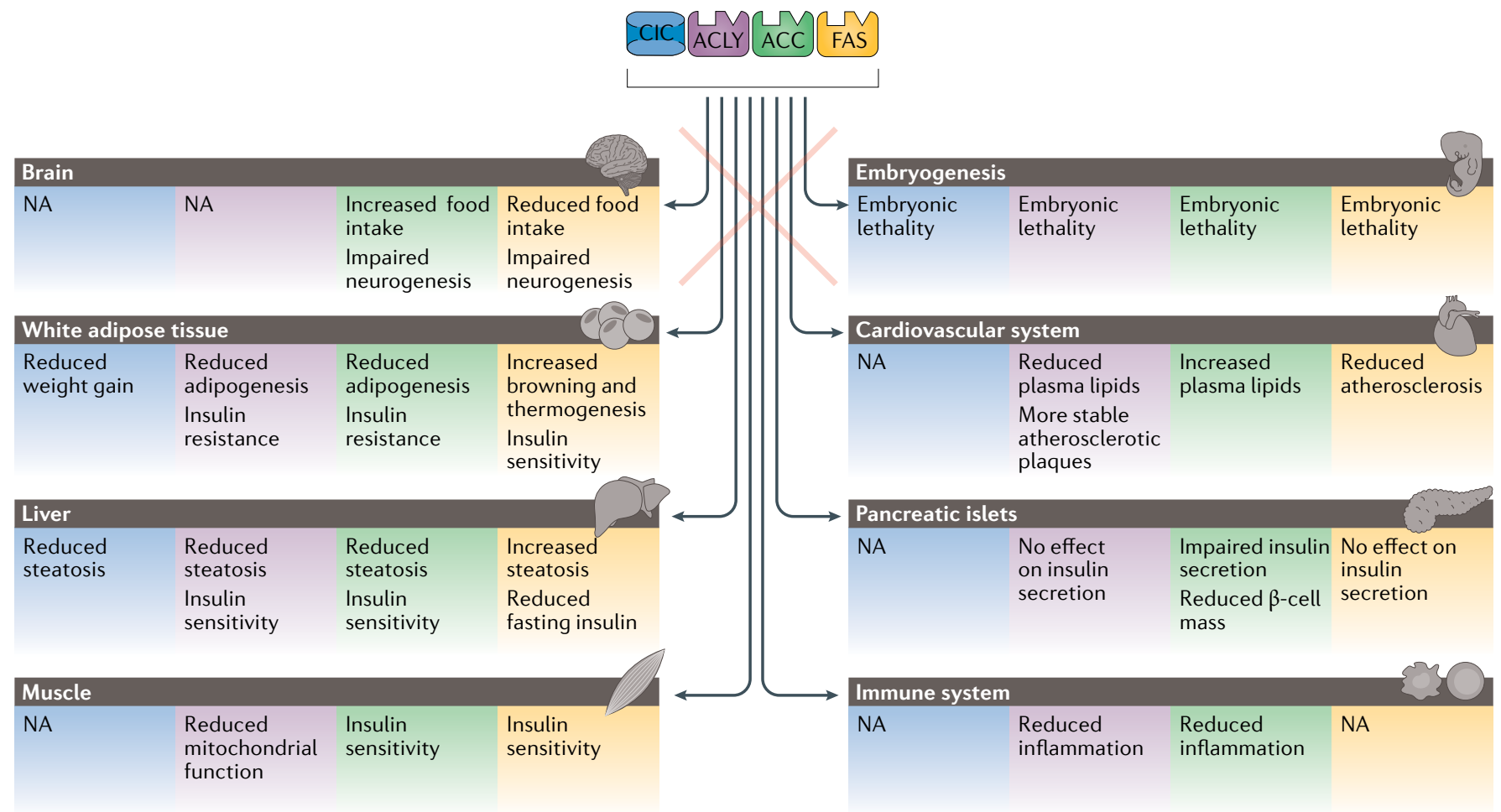

Fig. 2 | Tissue-specific actions of DNL. Important insights into distinct actions of citrate/isocitrate carrier (CIC) and de novo lipogenesis (DNL) enzymes in various tissues have been postulated from studies that employed animal models that lack one of these core components of the DNL pathway. The actions of each enzyme is colour coded: CIC-mediated effects are in blue boxes, ATP-citrate lyase (ACLY) in purple boxes, acetyl-CoA carboxylase (ACC) in green boxes and fatty acid synthase (FAS) in yellow boxes. NA, not available.

atherosclerotic plaques of mice and humans, suggesting that inhibition may be beneficial. Consistent with this concept, genetic inhibition of $\mathrm{Acly}^{74}$ or $\mathrm{Fas}^{76}$ within macrophages of ApoE mice reduces atherosclerosis. Surprisingly, there have been no studies examining the effects of genetic deletion of macrophage ACC on atherosclerosis.

\section{Cancer}

DNL is constitutively active in many cancer cells and contributes most of the intracellular lipid mass ${ }^{77}$. It is now well recognized that common genetic mutations (for example, in the gene encoding p53 $\left(\mathrm{REF}^{78}\right)$ or the gene encoding $\mathrm{PTEN}^{79}$ ) and growth factor signalling (epidermal growth factor ${ }^{80}$, HER2 $\left(\right.$ REF $\left.^{81}\right)$ and keratinocyte growth actor $^{82}$, ERK1/ERK2 MAPKs ${ }^{82,83}$ ) enhance the activity of SREBP1 leading to upregulation of lipogenic genes. In addition to transcriptional control, enhanced glucose uptake - common in glycolytic tumours increases citrate availability, which allosterically activates ACLY and ACC. Mutations in PTEN, which lead to constitutive activation of AKT, also increase the activity of ACLY through phosphorylation at Ser454, while at the same time enhancing ACC activity by suppressing $\mathrm{AMPK}^{84}$. Similarly, mutations in LKB1 reduce activating AMPK phosphorylation, thereby increasing ACC activity and cell proliferation ${ }^{85}$. Thus, tumours use multiple overlapping mechanisms to enhance DNL.

Genetic evidence that supports a crucial role for DNL enzymes in regulating tumorigenesis has been obtained from multiple studies involving knock-down of $\mathrm{ACLY}^{7,86,87}, \mathrm{ACC}^{88}$ and $\mathrm{FAS}^{89,90}$ in a wide variety of distinct tumour types (reviewed in REFS ${ }^{91,92}$ ). Inhibition of DNL also increases sensitivity to standards of care such as radiation ${ }^{93}$, androgen deprivation ${ }^{94}$ chemotherapies or tyrosine kinase inhibitors such as sorafenib ${ }^{95}$. Survival analysis examining the relationship between DNL-expressing genes also supports an important relationship across different tumour types ${ }^{96}$. These data suggest that inhibiting DNL may exert favourable effects in multiple types of cancer.

\section{Infection and immunity}

Inflammation and metabolism are intimately linked, as the maintenance of cellular defence systems and removal of pathogens is an energetically demanding process. Upon activation, immune cells undergo metabolic reprogramming to meet the energy demand for cell proliferation, differentiation and cytokine production ${ }^{97}$. One of the metabolic switches that occurs during these processes is the induction of $\mathrm{DNL}^{98,99}$. It is known that immune cells that subsume a pro-inflammatory role (M1-like) use aerobic glycolysis and have high rates of DNL, whereas anti-inflammatory immune cells (M2-like) use predominantly oxidative metabolism and have low rates of DNL. Although this is an oversimplification of an extremely complicated non-binary relationship, it does provide a general context for understanding the link between inflammation and metabolism ${ }^{100}$. The triggers for immune activation vary, but findings in 
Metabolic diseases

A group of diseases

characterized by disrupted

metabolism that adversely

affect normal cellular function

and structure. chronic metabolic diseases such as obesity, NASH and CVD have found an important role for microbial products such as lipopolysaccharide (LPS) and ectopic lipid accumulation (cholesterol and fatty acids) ${ }^{101}$.

Both ACC and ACLY regulate immune function. The inhibition of ACLY inhibits LPS-induced inflammation ${ }^{102}$ in some but not all studies ${ }^{74}$ and is important for mediating the anti-inflammatory effects of IL-4 (REF. ${ }^{103}$ ) and IL-2 (REF. ${ }^{104}$ ). In human and mouse naive T cells, deletion of ACC1 restrains the development of pro-inflammatory $\mathrm{T}$ helper $17\left(\mathrm{~T}_{\mathrm{H}} 17\right)$ cells and promotes the formation of anti-inflammatory regulatory $\mathrm{T}$ cells; a finding that translates in vivo into the attenuation of $\mathrm{T}_{\mathrm{H}} 17$-mediated autoimmune disease $\mathrm{e}^{98}$ and infection-associated intestinal inflammation ${ }^{105}$. ACC1 is also crucial in obesity-induced $\mathrm{T}_{\mathrm{H}} 17$ cell differentiation in humans ${ }^{12}$. Deletion of ACC 1 also reduces antigen-specific clonal expansion of cytotoxic $\mathrm{CD}^{+} \mathrm{T}$ cell populations during Listeria infection ${ }^{106}$. Interestingly, this increase in DNL is exploited by many viruses for the formation of their replication complex, and as a result, inhibition of SREBP, ACLY, ACC1 and FAS reduces viral replication ${ }^{9,10,107}$. These studies suggest that inhibiting DNL may be effective at reducing inflammation in chronic disease settings such as obesity and NASH and may exert positive effects to reduce viral replication. However, whether this may be exploited therapeutically without suppressing host immunity, which requires activation of DNL to promote an M1 phenotype, remains to be determined.

\section{Neurogenesis}

Neural differentiation occurs throughout life $\mathrm{e}^{108}$ and is strongly associated with upregulation of DNL in neural stem and progenitor cells ${ }^{14}$. The inactivation of FAS and ACC reduces neurodifferentiation ${ }^{14}$, and this has been linked to neurodegenerative diseases, including multiple sclerosis ${ }^{109}$, Parkinson disease ${ }^{110}$ and Alzheimer disease $^{111}$. Interestingly, individuals with a variant of FAS (FAS-R1819W) have cognitive disorders ${ }^{112}$, a finding that has been shown to be consistent in rodents overexpressing this variant ${ }^{113}$. In addition to neural stem and progenitor cells, DNL is central for the proper morphological formation and function of neurons. FAS is highly expressed in neurons and is crucial for dendrite branching and function ${ }^{114}$. DNL is also essential for myelination, as lipids comprise a large portion of the myelin membrane ${ }^{115}$. Recent studies have demonstrated that FAS is necessary for the correct onset of myelination and proper myelin growth by Schwann cells in the peripheral nervous system ${ }^{116}$ and oligodendrocytes in the central nervous system ${ }^{109}$. These data suggest that inhibition of DNL in the nervous system, not only in fetal development but also in adulthood, could potentially promote the progression of neurodegenerative disease.

\section{CIC}

\section{Regulation and structure}

CIC is encoded by the SLC25A1 gene and is ubiquitously expressed, with highest expression in liver, reproductive organs, gastrointestinal tract and adipose tissue (see Related links). It is located within the inner mitochondrial membrane, and primarily catalyses the efflux of tricarboxylates such as citrate and isocitrate in exchange for tricarboxylates, dicarboxylates and phosphoenolpyruvate ${ }^{117}$. LCFA-CoAs inhibit CIC in a reversible manner, competitive with citrate ${ }^{118}$, while acetylation can increase allosteric activation by citrate ${ }^{119}$ (FIG. 3).

Little is known about how the structural components of CIC are implicated in physiological regulation of activity. Structurally, eukaryote CIC is composed of three homologous domains, each of which form two hydrophobic membrane-spanning $\alpha$-helices that are connected by hydrophilic loops that span from the intermembrane space to the mitochondrial matrix ${ }^{117}$ (FIG. 4). There are at least two citrate binding sites, residing at different depths within the membrane bilayer and serving as the binding site of CIC inhibitors. Residues within sites 1 and 2 form six and eight hydrogen bonds with citrate, respectively ${ }^{120}$. CIC site 1 is kinetically accessible to anions from the inner surface and determines specificity to internal substrate as it moves through the $\mathrm{CIC}^{121}$. After binding to site 1 , citrate is transferred to site 2 , before being released into the intermembrane space where it then diffuses through a voltage-dependent anion transport channel within the mitochondrial outer membrane, into the cytoplasm.

\section{Pharmacological inhibitors}

The first-generation CIC inhibitor to have a higher affinity for the transporter than any substrate was benzenetricarboxylate (BTC) $)^{117}$ (TABLE 1; Supplementary Table 1), which inhibits the CIC in a mixed competitive and uncompetitive manner ${ }^{117,122}$. BTC is structurally similar to citrate and primarily interacts with citrate binding site 2 (REF. ${ }^{122}$ ) (FIG. 4). Although this inhibitor has been widely used for structural and functional characterization of the CIC, potential binding of the inhibitor to other citrate binding proteins has limited its therapeutic development.

CTPI-1, also known as compound 792949, is a next-generation competitive CIC inhibitor identified via in silico screening of commercially available small molecules. It has a slightly higher affinity for CIC than BTC does and binds to residues from both citrate binding sites simultaneously ${ }^{122}$. This inhibitor was identified in yeast CIC and later it was found to exhibit suboptimal binding for human CIC, potentially owing to a key amino acid difference in the citrate binding sites between the species ${ }^{123}$.

Attempts to optimize compounds specific for human CIC led to the identification of CTPI-2, which exhibited a 20 -fold improvement in binding affinity relative to CTPI- 1 and inhibited citrate transport at lower concentrations $^{123}$. Furthermore, it has shown several favourable effects in preclinical models as discussed below, indicating the potential of the inhibitor for development.

\section{Therapeutic indications}

Metabolic diseases. Lowering cytosolic citrate would be expected to inhibit DNL by reducing both substrate availability and allosteric activation of ACLY and $\mathrm{ACC}^{124,125}$. Consistent with this concept, BTC 
lowers triglyceride content in primary hepatocytes ${ }^{126}$ (Supplementary Fig. 1). CIC expression is increased in the liver of people with NASH, and CTPI-2 treatment of HFD-fed mice reversed steatohepatitis and liver injury, with a concomitant reduction in serum cholesterol and triglycerides ${ }^{43}$. In addition, CTPI-2 reduced fasting glucose and normalized glucose tolerance and insulin sensitivity, potentially through targeting gluconeogenesis. However, as CTPI-2 also lowered body mass through mechanisms that are not understood, this may have

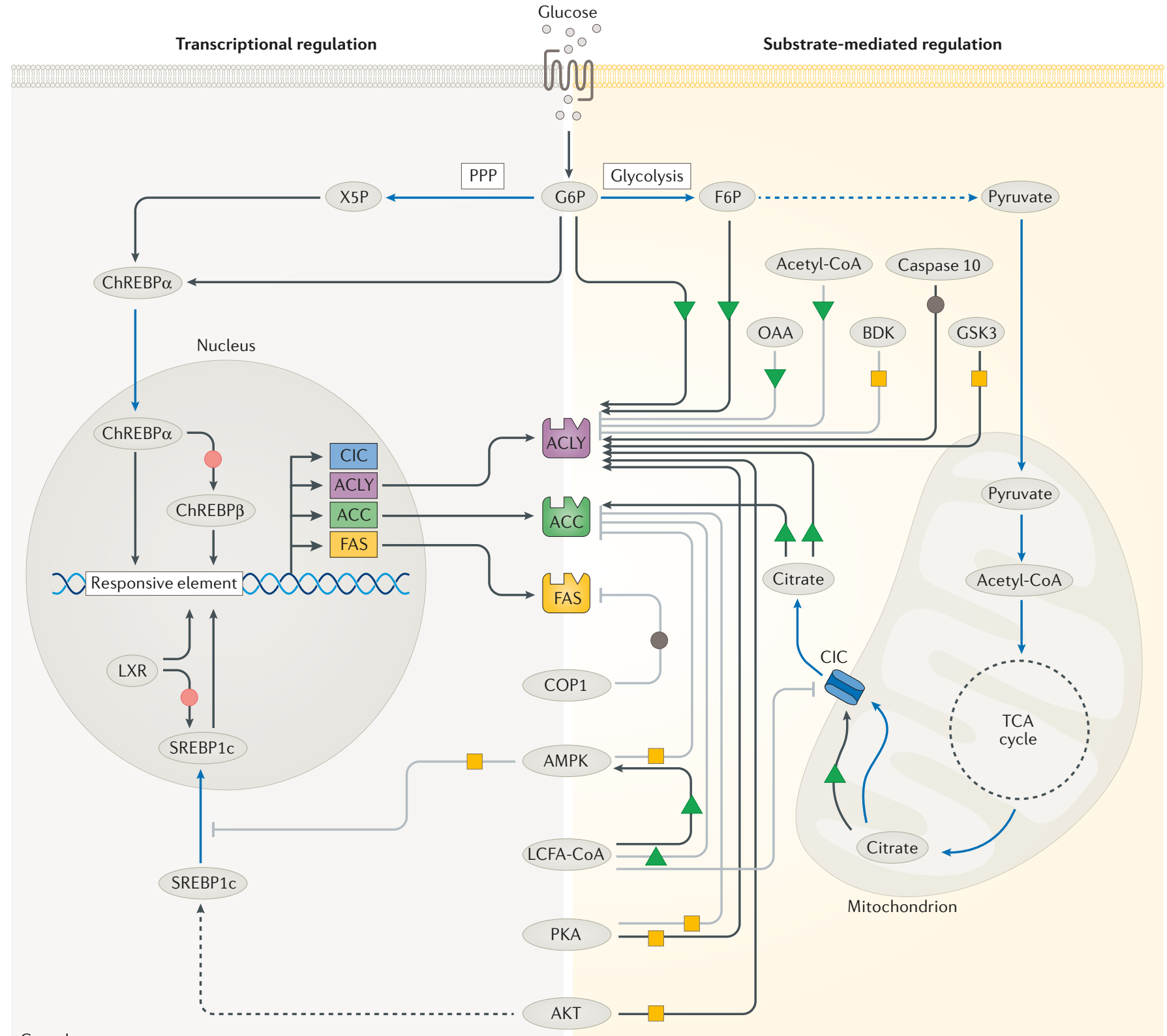

Cytoplasm

$\begin{array}{llll}\text { Allosteric regulation } & \text { Transcription } & \text { - Direct interaction } & \text { Activation } \\ \text { Phosphorylation } & \text { Degradation } & \text {-..-. Indirect interaction } & \text { - Pathway }\end{array}$

Fig. 3 | Physiological regulation of DNL. Regulatory mechanisms of de novo lipogenesis (DNL) involve allosteric regulation, covalent modifications and transcriptional changes. Allosteric activators include citrate, glucose 6-phosphate (G6P) and fructose 6-phosphate (F6P) while oxaloacetate (OAA) and long-chain fatty acyl (LCFA)-CoAs are allosteric inhibitors. Regulatory phosphorylation is facilitated by several enzymes including AMP-activated protein kinase (AMPK), AKT, branched-chain $\alpha$-keto dehydrogenase kinase (BDK), glycogen synthase kinase 3 (GSK3) and protein kinase $\mathrm{A}(\mathrm{PKA})$, whereas caspase 10 and constitutive photomorphogenic 1
(COP1) facilitate the degradation of ATP-citrate lyase (ACLY) and fatty acid synthase (FAS), respectively. Transcriptional modifications are regulated by two major transcription factors, sterol regulatory element-binding protein 1c (SREBP1c) and carbohydrate-responsive element-binding protein (ChREBP). Additional transcription factors, such as liver X receptor (LXR) are also implicated in the transcriptional regulation to varying degrees of importance depending on the cell type. ACC, acetyl-CoA carboxylase; $\mathrm{CIC}$, citrate/isocitrate carrier; FAS, fatty acid synthase; PPP, pentose phosphate pathway; TCA, tricarboxylic acid; X5P, xylulose 5-phosphate. 
a $\mathrm{CIC}$ inhibitors

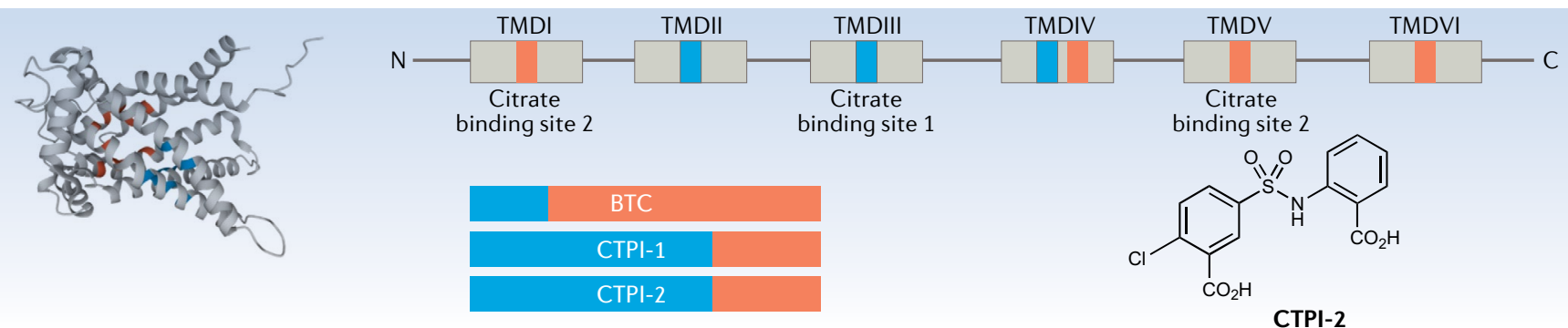

\section{b ACLY inhibitors}
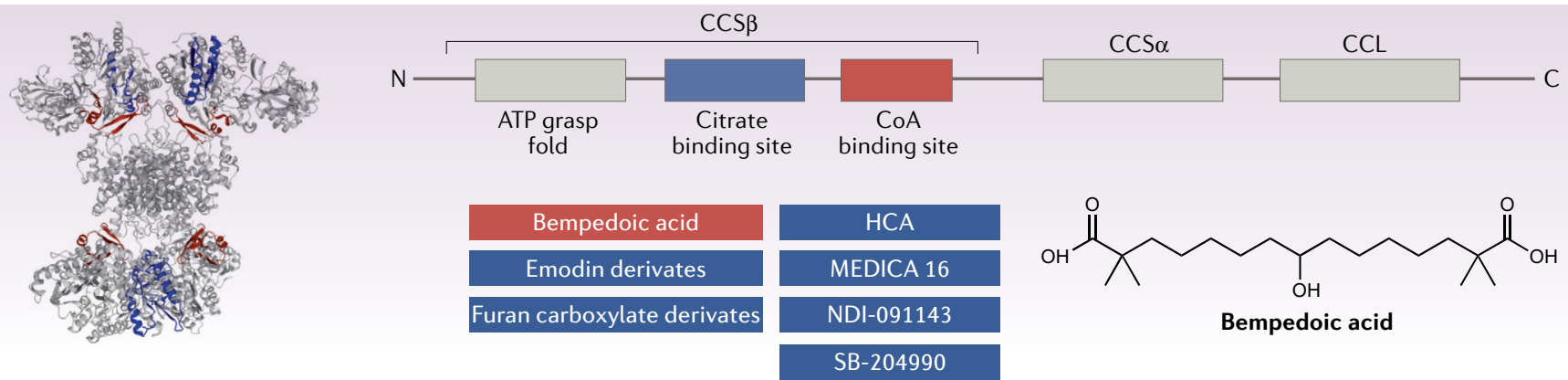

\section{c ACC inhibitors}
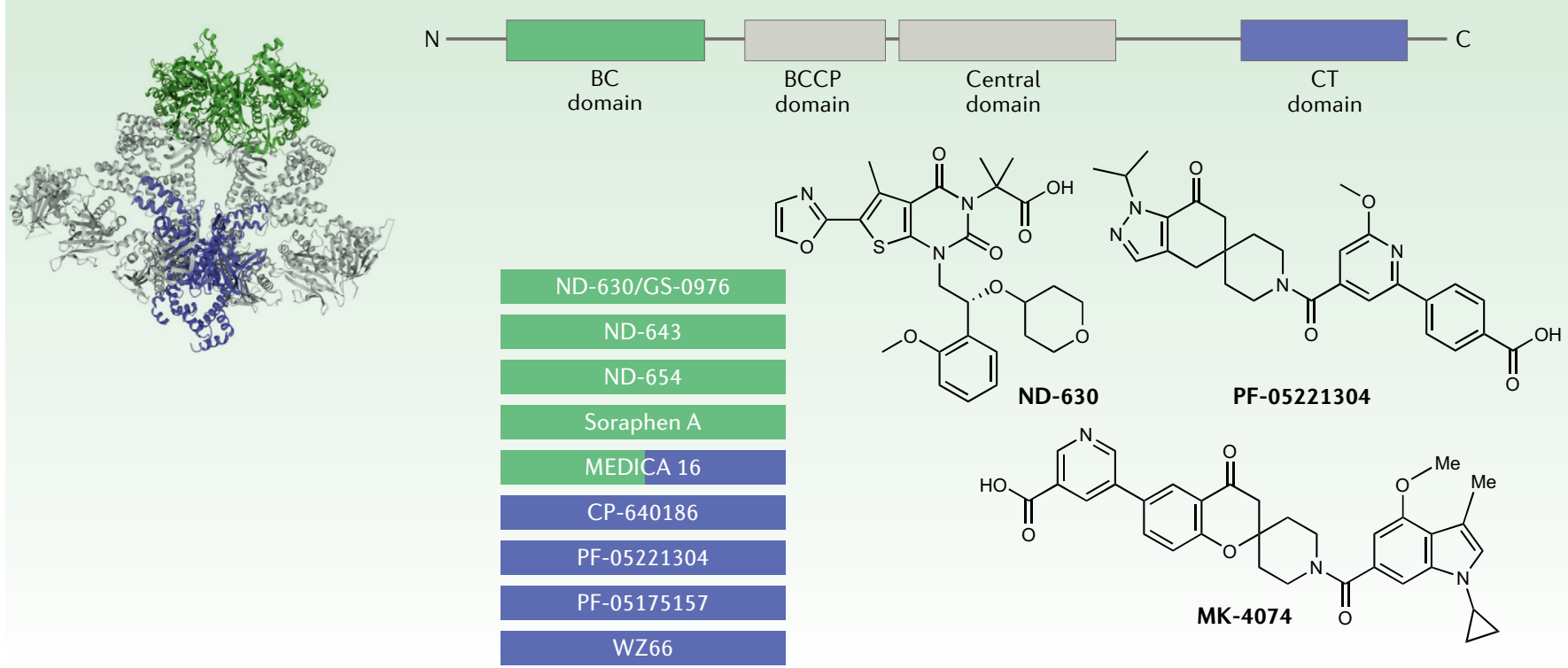

\section{d FAS inhibitors}
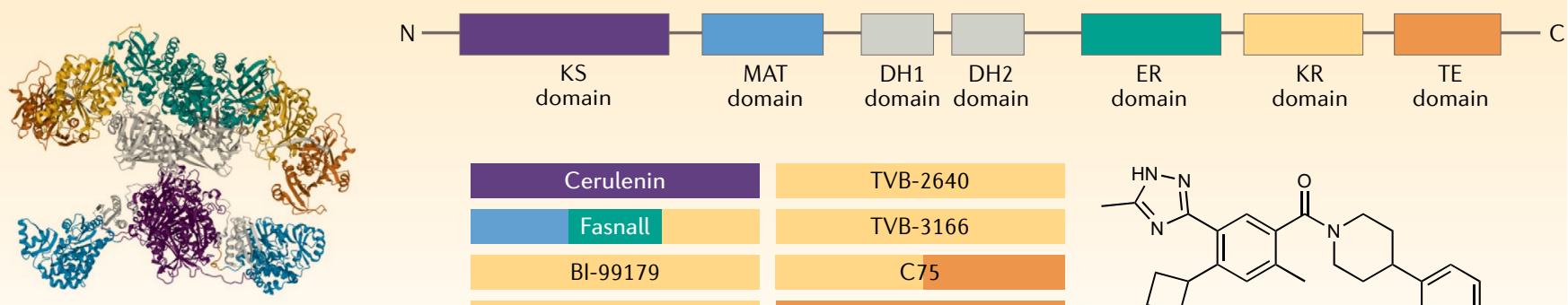

\begin{tabular}{|c|}
\hline Cerulenin \\
\hline Fasnall \\
\hline BI-99179 \\
\hline FT-4101 \\
\hline GSK837149A \\
\hline GSK2194069 \\
\hline
\end{tabular}

\begin{tabular}{|c|}
\hline TVB-2640 \\
\hline TVB-3166 \\
\hline C75 \\
\hline MP-ML-24-N1 \\
\hline IPI-9119 \\
\hline Orlistat \\
\hline
\end{tabular}<smiles>Cc1nc(-c2cc(C(=O)N3CCC(c4ccc(C#N)cc4)CC3)c(C)cc2C2CCC2)n[nH]1</smiles> 
4 Fig. 4 | Structural domains and binding sites of DNL inhibitors with chemical structures of the most advanced inhibitors. Lipogenesis inhibitors interact with one or more druggable sites of the enzyme to exhibit an inhibitory effect. Linear organization and model representation of each enzyme are shown with known inhibitor binding sites in colours. Inhibitors with known enzyme binding sites are colour coded with their respective interaction sites. a | Citrate/isocitrate carrier (CIC) inhibitors: compounds that bind to citrate binding site 1 are shown in light blue and those that bind to citrate binding site 2 in orange. The chemical structure of CTPI- 2 is shown. b | ATP-citrate lyase (ACLY) inhibitors: compounds that interact with the CoA binding site are highlighted in red and those that interact with citrate binding site in dark blue. The chemical structure of bempedoic acid is shown. $\mathbf{c}$ Acetyl-CoA carboxylase (ACC) inhibitors: compounds that target the biotin carboxylase (BC) domain are highlighted in green and those that target the carboxyl transferase (CT) domain are highlighted in violet. Chemical structures of clinical stage inhibitors are shown. $\mathbf{d}$ | Fatty acid synthase (FAS) inhibitors: inhibitors that bind to the $\beta$-ketoacyl synthase (KS) domain are highlighted in purple, inhibitors that bind to malonyl-acetyl transferase (MAT) are in blue, inhibitors that bind to enoyl reductase $(E R)$ are in green, inhibitors that bind to $\beta$-ketoreductase $(K R)$ in yellow and those that bind to thioesterase (TE) in orange. The chemical structure of TVB-2640 is shown.

$\mathrm{BCCP}$, biotin carboxyl carrier protein; BTC, benzenetricarboxylate; $\mathrm{CCL}$, citryl-CoA lyase; CCS, citryl-CoA synthetase; $\mathrm{DH}$, dehydratase; HCA, (-)-hydroxycitric acid; TMD, transmembrane domain. Part a CIC structure adapted from P53007, CC BY 4.0; part b ACLY structure adapted from PDB ID 6POF, CC BY 1.0; part c ACC structure adapted from PDB ID 5CSK, CC BY 1.0; part d FAS structure adapted from PDB ID 2VZ8, CC BY 1.0.

contributed to improvements in glucose homeostasis. BTC acutely inhibits glucose-stimulated insulin secretion in INS1 cells and islets ${ }^{127}$; however, in vivo effects on pancreatic islets have not been evaluated. These data suggest that inhibition of CIC may exert favourable effects on obesity, NAFLD and T2D. Future studies are needed to determine the mechanisms that contribute to weight loss and whether this is required for the beneficial effects on liver steatosis and glucose homeostasis.

One potential mechanism by which CIC inhibitors may exert positive effects in metabolic disease independently of reductions in body mass, may involve inhibition of inflammation. Inflammatory stimuli such as LPS, TNFa and IFN $\gamma$, elicit metabolic reprogramming in macrophages and natural killer cells that leads to increases in CIC expression, suggesting that inhibition may exert anti-inflammatory effects ${ }^{128,129}$. In cultured macrophages, CTPI-1 inhibits inflammatory responses induced by TNF $\alpha$ and IFN $\gamma^{128}$. Similarly, in obese mice CTPI-2 blocks inflammatory macrophage infiltration into the liver and adipose tissue and this is accompanied by a decrease in circulating pro-inflammatory

Table 1 | CIC inhibitors

\begin{tabular}{|c|c|c|c|}
\hline $\begin{array}{l}\text { Compound } \\
\text { (developer) }\end{array}$ & $\begin{array}{l}\text { Potency in } \\
\text { biochemical } \\
\text { assays }\end{array}$ & $\begin{array}{l}\text { Indication and/or preclinical } \\
\text { effects }\end{array}$ & Refs \\
\hline \multicolumn{4}{|l|}{ Preclinical stage inhibitors } \\
\hline Benzenetricarboxylate & $K_{\mathrm{i}}: 0.07-0.16 \mathrm{mM}$ & $\begin{array}{l}\text { Decreased triglyceride } \\
\text { synthesis, reduced glucose- } \\
\text { stimulated insulin secretion and } \\
\text { inhibited cancer cell growth }\end{array}$ & $\begin{array}{r}126, \\
127,132\end{array}$ \\
\hline $\begin{array}{l}\text { CPTI-1 (Rosalind Franklin } \\
\text { University of Medicine } \\
\text { and Science, USA) }\end{array}$ & $\begin{array}{l}K_{i}: \\
0.048-0.07 \mathrm{mM}\end{array}$ & $\begin{array}{l}\text { Reduced inflammation and } \\
\text { inhibited cancer cell growth }\end{array}$ & 43,128 \\
\hline $\begin{array}{l}\text { CPTI-2 (Lombardi } \\
\text { Comprehensive Cancer } \\
\text { Center, USA) }\end{array}$ & $K_{d}: 3.5 \mu \mathrm{M}$ & $\begin{array}{l}\text { Decreased hepatic steatosis, } \\
\text { improved lipid and glucose } \\
\text { homeostasis, and inhibited } \\
\text { cancer cell growth }\end{array}$ & 43,129 \\
\hline
\end{tabular}

cytokines $^{43}$. These data suggest that inhibiting CIC may reduce inflammation and this may exert a positive effect on metabolic diseases.

Cancer. As CIC expression is increased in several different cancer cells ${ }^{130}$ and inhibition of CIC blunts cell growth ${ }^{131}$, CIC has emerged as an attractive target for anticancer drug development. All three CIC inhibitors have been found to exert anticancer activity in cultured cancer cell lines ${ }^{123,132,133}$ and reduce tumour growth in vivo ${ }^{132,133}$. Future studies are needed to evaluate the effects of these inhibitors in clinical settings.

\section{ACLY}

\section{Regulation and structure}

ACLY expression is highest in adipose and liver and lowest in skeletal muscle (see Related links). Although ACLY is predominantly a cytosolic enzyme, it is also found within the nucleus (see Related links). The activation of ACLY involves four steps: first, Mg-ATP binding, and phosphorylation of His760, which catalyses the formation of an enzyme-bound citryl-phosphate, followed by a CoA attack and formation of a citryl-CoA intermediate and finally, citryl-CoA cleavage into final products acetyl-CoA and oxaloacetate (OAA). This reaction is allosterically activated by the glycolytic intermediates glucose 6-phosphate (G6P) and fructose 6-phosphate (F6P), with the latter being more potent ${ }^{134}$ (FIG. 3). Citrate also allosterically activates ACLY while the products of the reaction, acetyl-CoA and OAA, inhibit enzyme activity ${ }^{124}$.

In addition to allosteric activation, early studies identified that insulin ${ }^{135}$ and glucagon ${ }^{136}$ increase Ser/Thr phosphorylation on ACLY (FIG. 3), with subsequent studies establishing that Thr446 and Ser450 are phosphorylated by glycogen synthase kinase 3 (GSK3) ${ }^{137}$, and Ser454 is phosphorylated by protein kinase A (PKA) ${ }^{136}$ and the 'insulin-stimulated kinase ${ }^{\text {'138 }}$ that was subsequently identified as AKT (also known as PKB) ${ }^{139}$. mTORC2 was also suggested to phosphorylate ACLY Ser454 (REF. ${ }^{8}$ ), an effect shown to require $\mathrm{AKT}^{140}$. More recently, branched-chain ketoacid dehydrogenase kinase was also found to phosphorylate Ser454, thus linking ACLY activity with amino acid availability and insulin resistance ${ }^{141}$. Although initial studies found that Ser454 phosphorylation had minimal effects on ACLY activity ${ }^{142}$, further work established that phosphorylation of this residue decreased sensitivity to allosteric activation by F6P and G6 $\mathrm{P}^{134}$. Subsequent studies in various cell lines have confirmed the important effects of this phosphorylation event in enhancing ACLY activity ${ }^{8,104,143}$. In addition, ACLY is regulated by cleavage of the enzyme at a post-transcriptional level ${ }^{144}$.

The structural underpinnings of the chemical reactions mediated by ACLY are still not fully understood $^{124,145}$. ACLY consists of two modules, ACLY-A and ACLY-B, which are present as separate domains (heteromeric) in prokaryotes, but are linked (homomeric) in animals $^{146}$ to form a functional tetrameric structure ${ }^{124}$ (FIG. 4). Each monomer consists of an N-terminal citryl-CoA synthetase (CCS) module, containing CCS $\beta$ and CCSa regions, and a C-terminal citryl-CoA lyase (CCL) domain ${ }^{145}$. The ACLY-A module forms the CCS $\beta$ 
Table 2 | ACLY inhibitors

\begin{tabular}{|c|c|c|c|}
\hline Compound (developer) & $\begin{array}{l}\text { Potency in } \\
\text { biochemical assays }\end{array}$ & $\begin{array}{l}\text { Indication and/or preclinical } \\
\text { effects }\end{array}$ & $\begin{array}{l}\text { Clinical trial ID } \\
\text { or refs }\end{array}$ \\
\hline \multicolumn{4}{|l|}{ Clinical stage inhibitors } \\
\hline $\begin{array}{l}\text { Bempedoic acid (Esperion } \\
\text { Therapeutics, USA) }\end{array}$ & $K_{i}: 2 \mu \mathrm{M}$ & $\begin{array}{l}\text { Primary hypercholesterolaemia } \\
\text { and established atherosclerotic } \\
\text { cardiovascular disease }\end{array}$ & Approved \\
\hline \multirow[t]{2}{*}{ Hydroxycitrate } & \multirow[t]{2}{*}{$K_{\mathrm{i}}: 0.15 \mu \mathrm{M}$} & Obesity and type 2 diabetes & $\begin{array}{l}\text { NCT01238887 } \\
\text { and NCT00699413 } \\
\text { (terminated, } \\
\text { phases I and IV) }\end{array}$ \\
\hline & & Urine chemistries & $\begin{array}{l}\text { NCT03348228 } \\
\text { (in progress) }\end{array}$ \\
\hline \multicolumn{4}{|l|}{ Preclinical stage inhibitors } \\
\hline $\begin{array}{l}\text { BMS-303141 (Bristol-Myers Squibb } \\
\text { Pharmaceutical Research Institute, } \\
\text { USA) }\end{array}$ & $\mathrm{IC}_{50}: 0.13 \mu \mathrm{M}$ & $\begin{array}{l}\text { Reduced weight gain, plasma lipids } \\
\text { and glycaemia, and inhibited cancer } \\
\text { cell growth }\end{array}$ & 94,160 \\
\hline $\begin{array}{l}\text { Emodin derivates (Harvard } \\
\text { Medical School, USA) }\end{array}$ & $\mathrm{IC}_{50}: 3-30 \mu \mathrm{M}$ & Inhibited cancer cell growth & 155 \\
\hline $\begin{array}{l}\text { Furan carboxylate derivates } \\
\text { (Harvard Medical School, USA) }\end{array}$ & $\mathrm{IC}_{50}: 4.1-11.9 \mu \mathrm{M}$ & Inhibited cancer cell growth & 159 \\
\hline $\begin{array}{l}\text { MEDICA } 16 \text { (Hadassah Medical } \\
\text { School, Israel) }\end{array}$ & $K_{\mathrm{i}}: 16 \mu \mathrm{M}$ & $\begin{array}{l}\text { Reduced weight gain, hepatic } \\
\text { steatosis, plasma lipids and } \\
\text { atherosclerosis }\end{array}$ & $162,177,178$ \\
\hline $\begin{array}{l}\text { SB-204990 (SmithKline Beecham } \\
\text { Pharmaceuticals, UK) }\end{array}$ & $K_{\mathrm{i}}: 1 \mu \mathrm{M}$ & $\begin{array}{l}\text { Lowered plasma lipids and inhibited } \\
\text { tumour growth }\end{array}$ & 86,158 \\
\hline \multicolumn{4}{|l|}{ Discovery stage inhibitor } \\
\hline $\begin{array}{l}\text { NDI-091143 (Nimbus Therapeutics, } \\
\text { USA) }\end{array}$ & $K_{\mathrm{i}}: 0.07 \mu \mathrm{M}$ & No functional studies reported & 161 \\
\hline
\end{tabular}

region, while ACLY-B forms the remaining domains of the monomer, with Ser/Thr phosphorylation occurring within a linker region between the modules ${ }^{145}$. Recent studies have provided a detailed structural basis for the multistep catalytic mechanism of ACLY, showing that citrate binds to the CCS $\beta$ region, while the CCS $\alpha$ region serves as a CoA binding site ${ }^{124}$. An additional CoA binding site is in the CCL domain ${ }^{124,145}$; however, it is controversial whether binding at this site is required for acetyl-CoA formation.

\section{Pharmacological inhibitors}

ACLY is an attractive drug target owing to its strategic position at the nexus of fatty acid, cholesterol and carbohydrate metabolism. As such, several ACLY inhibitors have been identified on the basis of their ability to mediate concomitant inhibition of fatty acid and cholesterol synthesis, while activating fatty acid $\beta$-oxidation.

The first identified and extensively studied inhibitor of ACLY was (-)-hydroxycitric acid (HCA), a derivative of citric acid found in tropical plants Garcinia cambogia and Hibiscus subdariff $a^{147}$. HCA inhibits ACLY by competing with citrate ${ }^{147}$, which results in concomitant suppression of DNL and cholesterol biosynthesis ${ }^{148}$ (TABLE 2; Supplementary Table 2). However, HCA possesses poor physicochemical properties and was later found to allosterically activate ACC $^{149}$. Although numerous attempts were made to improve on the HCA scaffold, these efforts were unsuccessful as off-target effects continued to be observed ${ }^{150}$.
Additional screening of natural products identified several other ACLY inhibitors, including purpurone ${ }^{151}$, a series of anthrones and anthraquinones derived from Penicillium sp. ${ }^{152}$, radicicol, a 14 -membered macrolide originally isolated from Monosporium bonorden ${ }^{153}$, and cucurbitacin B, a natural bioactive compound abundant in cucumber ${ }^{154}$. Another series of inhibitors was developed on the chemical scaffold of the natural product emodin ${ }^{155}$. Although many of these compounds suppressed ACLY activity in biochemical assays, their specificity for ACLY and mechanisms of action remained unresolved $^{156}$

Several inhibitors were designed on the basis of an alternative targeting strategy aimed to disrupt the formation of a stable citryl-CoA intermediate bound to the active site. SB-201076 was a promising compound that demonstrated activity against purified rat ACLY, but was inactive in cell-based DNL assays owing to poor cell permeability ${ }^{157}$. Improved cell permeability was achieved by generating the lactone prodrug analogue, SB-204990, which undergoes hydrolysis and activation once inside the cell to yield the active metabolite, SB-201076 (REF. ${ }^{158}$ ). However, development of this series was halted before clinical development. More recently, in silico screening identified four subtypes of furans and benzofurans that inhibit ACLY by also binding to the citrate binding domain ${ }^{159}$.

A novel chemical scaffold discovered by highthroughput screening (HTS) led to the identification of BMS-303141, a 2-hydroxy- $N$-arylbenzenesulfonamide ${ }^{160}$. 
This compound inhibits ACLY, but also ACC1 and ACC2, although nearly 100 -fold less potently ${ }^{160}$. Building on the BMS-303141 chemical scaffold, Nimbus Therapeutics used rational computer-aided design to develop a new series of ACLY inhibitors. The most potent compound, NDI-091143, inhibited human ACLY competitively with respect to citrate. The use of cryo-electron microscopy revealed an unexpected allosteric mechanism of inhibition whereby NDI-091143 bound next to the citrate binding site in a hydrophobic cavity, resulting in an extensive conformational change that prevented citrate binding to the enzyme. Although no cell-based or in vivo data were reported, the identification of this novel allosteric mechanism provides a new approach to discover novel ACLY inhibitors with improved drug-like properties ${ }^{161}$.

One of the first synthetic fatty acid-like ACLY inhibitors was MEDICA 16. This compound was designed by modifying long-chain dicarboxylic fatty acids to generate $\beta \beta^{\prime}$-methyl-substituted $\alpha, \omega$-dicarboxylic acids, with the aim of maintaining the lipid-regulating properties of natural long-chain fatty acids while improving drug-like properties by preventing $\beta$-oxidation and enzymatic esterification ${ }^{162}$. MEDICA 16 inhibited ACLY competitively with citrate ${ }^{163}$ and ACC competitively with acetyl-CoA and ATP ${ }^{164}$. A similar dicarboxylic acid, 3-thiadicarboxylic acid, was synthesized by replacing the dimethyl substitution in the $\beta$-position with a sulfur atom. This molecule inhibited ACLY and FAS, resulting in reduced levels of plasma triglycerides and cholesterol ${ }^{165}$. However, neither molecule advanced beyond preclinical studies.

Using a phenotypic screen based on inhibiting fatty acid and sterol synthesis in primary rat liver cells, Esperion Therapeutics discovered a liver-specific inhibitor, ETC1002, 8-hydroxy-2,2,14,14-tetramethylpentadecanedioic acid, also known as bempedoic acid and ESP-55016 $\left(\right.$ REF $\left.^{166}\right)$. Studies have established that bempedoyl-CoA potently inhibits recombinant human ACLY competitively with CoA and that the prodrug (bempedoic acid) is inactive ${ }^{167}$. Importantly, conversion into the CoA conjugate is dependent on very long-chain acyl-CoA synthetase (ACSVL1/FATP2/SCL27A2), an enzyme highly expressed in the liver, but not in most other tissues including skeletal muscle, thus enabling the liver-specific actions of bempedoic acid ${ }^{167}$. And while bempedoyl-CoA also inhibits ACC $^{166}$ and activates AMPK $\beta 1$-containing heterotrimers ${ }^{167}$, the relevance of these activities has not been demonstrated, since potency towards ACC is relatively weak and bempedoic acid continues to suppress liver DNL even in the absence of the AMPK $\beta 1$ isoform ${ }^{167}$.

\section{Therapeutic indications}

Obesity. Preclinical studies in rodents found that HCA reduces body mass through a mechanism related to caloric restriction ${ }^{168,169}$, but this effect is not observed in humans ${ }^{170,171}$ (Supplementary Fig. 1). Bempedoic acid and BMS-303141, two of the better-characterized ACLY inhibitors, have strengthened a potential connection between weight loss and ACLY, with both reducing body weight gain and adiposity independently of changes in food intake in preclinical models ${ }^{160,166,167,172}$. Importantly, recent evidence has emerged from pooled analyses of clinical trials that bempedoic acid elicits modest weight loss in humans ${ }^{173}$. Studies examining the potential mechanisms by which ACLY inhibitors exert weight loss are warranted.

NAFLD-NASH and type 2 diabetes. Liver lipids and insulin sensitivity are directly linked; as such there is a very close connection between NAFLD and T2D. MEDICA 16 reduced liver lipid content ${ }^{162}$, hepatic glucose production, and improved peripheral insulin sensitivity ${ }^{174,175}$ in several distinct rodent models of obesity-induced insulin resistance. Bempedoic acid also reduced hepatic triglycerides and markers of inflammation in $\mathrm{Ldll}^{-/-}$mice fed a diet high in fat and cholesterol. Importantly, the liver lipid-lowering effects of bempedoic acid are independent of liver AMPK activation ${ }^{167}$. In multiple mouse models, bempedoic acid also reduced fasting glucose, fasting insulin and glucose intolerance, suggesting improvements in insulin sensitivity ${ }^{172}$. Importantly, these effects appear to be translated to humans, as a meta-analysis of randomized trials suggests that bempedoic acid reduces new incidence or worsening of diabetes ${ }^{176}$. Whether bempedoic acid is effective at reversing NASH and fibrosis remains to be determined.

Cardiovascular disease. Given its dual impact on cholesterol and fatty acid biosynthesis, pharmacological ACLY inhibition has been studied for CVD. Early studies in animal models demonstrated the broad lipid-lowering effects of MEDICA 16 (REFS ${ }^{177,178}$ ) on circulating levels of cholesterol and triglycerides and associated beneficial effects on vascular and myocardial lesions ${ }^{178}$. Studies of BMS-303141 and SB-204990 further support ACLY as a target for hyperlipidaemia, as both compounds effectively lowered circulating triglycerides and cholesterol in animal models of hyperlipidaemia ${ }^{158,160}$. Bempedoic acid also suppresses hepatic cholesterol and fatty acid biosynthesis ${ }^{172}$ and its hypolipidaemic actions have been demonstrated in hyperlipidaemic hamsters ${ }^{172}$, obese Zucker rats ${ }^{166}$ and in mice deficient for $\mathrm{ApoE}^{167}$ or the LDL receptor ${ }^{179}$ in which atherosclerosis is also reduced. In humans, bempedoic acid promotes dose-dependent LDL-cholesterol lowering effects as monotherapy, and when combined with a statin or ezetimibe ${ }^{180}$. Unlike rodents, in which bempedoic acid has a profound effect in reducing both plasma triglycerides and cholesterol, the primary effect of bempedoic acid in humans appears to be a reduction in plasma LDL-cholesterol ${ }^{180}$. The lack of effect on plasma triglycerides may potentially be due to lower liver DNL in humans compared with rodents. In addition to lowering LDL-cholesterol, bempedoic acid also reduces several plasma markers associated with atherosclerotic CVD such as total cholesterol, non-HDL cholesterol, plasma apoB, LDL particle numbers and high-sensitivity C-reactive protein ${ }^{180}$. More recently, bempedoic acid has been demonstrated to be a safe and effective therapeutic option for patients on maximally tolerated statin therapy ${ }^{181}$ or patients intolerant of statins ${ }^{182}$. Bempedoic acid was recently approved for patients with heterozygous familial 
hypercholesterolaemia (HeFH) (USA and Switzerland), established atherosclerotic CVD (ASCVD) (USA and Switzerland) who need additional LDL-cholesterol lowering, as an adjunct to diet and maximally tolerated statins (USA, EU, Switzerland and UK), and alone or with other lipid-lowering therapies in patients who are statin-intolerant or for whom a statin is contraindicated (EU and UK). The effect on cardiovascular morbidity and mortality has not been established.

Cancer. Substantial evidence supports the positive effects of pharmacological inhibition of ACLY using SB-204990 and BMS-303141 in cultured cancer cells ${ }^{94}$. However, despite the robust in vitro evidence, there is surprisingly few data supporting the concept that pharmacological inhibition of ACLY may be effective in vivo. For example, although early studies of HCA showed antitumour effects ${ }^{183}$, target specificity and suppression of food consumption and body mass confounded interpretation of the primary mechanism of action. Similarly, SB-204990 reduced tumour growth in several distinct xenograft models; however, weight loss was again a confounding variable and no direct effect on metabolic reprogramming within the tumour was reported ${ }^{86}$. More recently, bempedoic acid has been demonstrated to inhibit hepatocellular carcinoma in mice, but given the requirement for bempedoic acid to be converted into a CoA by ASCVL1, it is currently unclear whether this is due to direct tumour effects or is secondary to inhibition of ACLY in the liver ${ }^{87}$. Future studies in mouse models examining the effects of pharmacological ACLY inhibitors in conjunction with measures of target engagement within the tumour (for example, suppression of DNL) are needed to evaluate whether pharmacological inhibition of ACLY may be effective in cancer.

\section{ACC}

\section{Regulation and structure}

In mammals, there are two isoforms of ACC, ACC1 (also known as ACCa) and ACC2 (also known as ACC $\beta$ ). ACC1 is ubiquitously expressed (see Related links), whereas ACC2 is predominantly found in skeletal muscle, breast, adipose and liver (see Related links). ACC isoforms exhibit amino acid sequence similarity of $82 \%$ and $76 \%$ in their biotin carboxylase (BC) and carboxyl transferase (CT) domains, respectively ${ }^{184}$, with the major difference being an $\mathrm{N}$-terminal extension in ACC2 resulting in a higher molecular weight compared with ACC1 (REF. ${ }^{184}$ ). Although both ACC isoforms are expressed in the cytosol, it was hypothesized that the N-terminal extension in ACC2 might facilitate a preferential role (compared with ACC1) in controlling fatty acid oxidation, because of proximity to carnitine palmitoyltransferase 1 , which is allosterically inhibited by malonyl-CoA ${ }^{184}$. Genetic evidence supports significant overlap between ACC isoforms in regulating fatty acid oxidation and $\mathrm{DNL}^{47,49}$, suggesting that the relative importance of each isoform in regulating DNL and fatty acid oxidation is related to tissue-specific expression profiles rather than cellular localization.

The mechanisms linking tricarboxylic acid (TCA) cycle intermediates with increases in DNL were first uncovered in 1962 when it was found that citrate stimulated the activity of $\mathrm{ACC}^{125}$ (FIG. 3). Subsequent studies identified that activation was mediated by promoting polymerization of the enzyme, an effect inhibited by LCFA-CoAs ${ }^{185}$. In addition to allosteric control, ACC is inactivated by phosphorylation ${ }^{186}$, which is sufficient to overcome allosteric activation under physiological concentrations of citrate ${ }^{187}$ (FIG. 3). This suggested that phosphorylation, not allosteric control, may be the predominant mechanism regulating ACC activity. ACC is phosphorylated and inhibited by the AMPK at Ser79 $\left(\right.$ REF. $\left.^{188}\right)$. ACC2 activity is similarly inhibited by phosphorylation at a homologous site (Ser221). In hepatocytes, mutations in both ACC1 and ACC2 were necessary to exert maximal effects on DNL and fatty acid oxidation ${ }^{189}$. Interestingly, a recent study found that LCFA-CoAs directly activate AMPK ${ }^{190}$, thus enacting a bimodal mechanism involving both allosteric and covalent inhibition of ACC activity, to inhibit DNL and increase fatty acid oxidation.

The ACC enzyme consists of three domains - BC, biotin-containing carboxyl carrier protein (BCCP) and $\mathrm{CT}$ - which are assembled in a single chain in most eukaryotes including mammals (FIG. 4). In eukaryotes, ACC functions as dimers and higher oligomers with the $\mathrm{BC}$ and $\mathrm{CT}$ domain dimers located at the top and bottom of the structure, respectively, while the BCCP is located within the CT active site ${ }^{191}$. The ACC reaction involves two steps: the first is ATP-dependent carboxylation of a biotin moiety by the BCCP, followed by transfer of a carboxyl group from biotin to acetyl-CoA to produce malonyl-CoA, within the $\mathrm{CT}^{192}$. Recent structural studies have detailed the dynamic interactions that occur between polymerization state and filament structures upon exposure to citrate and palmitoyl-CoA, effectively locking the enzyme into catalytically competent or incompetent conformational states, respectively ${ }^{193}$. Phosphorylation at Ser79 in ACC1, and presumably also Ser221 in ACC2, also induces a large conformational change involving the $\mathrm{BC}$ dimer interface, which promotes dissociation of the dimer and inactivation ${ }^{194}$. Thus, both allosteric regulation of ACC by citrate and palmitoyl-CoA and phosphorylation by AMPK regulate enzyme activity through alterations in conformational state $^{188,193}$.

\section{Pharmacological inhibitors}

Inhibition of ACC lowers malonyl-CoA, which is also an allosteric inhibitor of carnitine palmitoyl transferase 1, the rate-limiting enzyme that controls the flux of fatty acids into the mitochondria for $\beta$-oxidation. Thus, ACC inhibition represents an attractive approach to simultaneously suppress DNL and increase fatty acid oxidation. Given the embryonic lethality associated with ACC1 inhibition, differential tissue-specific expression profiles and potential compensation by the alternative isozyme, isozyme-specific (ACC1 or ACC2) and nonspecific (ACC1 and ACC2) inhibitors, as well as tissue-selective inhibitors, have been pursued.

The first generation of ACC inhibitors to be studied were soraphen A and TOFA (5-(tetradecyloxy)2 -furancarboxylic acid). Soraphen A is a macrocyclic 
polyketide, originally isolated from the soil myxobacterium Sorangium cellulosum for its potent antifungal activity. It was later identified as an inhibitor of yeast and rat ACC1 (REF. ${ }^{195}$ ). In eukaryotes soraphen A binds to the $\mathrm{BC}$ domain, allosterically inhibiting enzyme activity by disrupting oligomerization; however, this does not occur in prokaryotes owing to large structural differences within the BC domain ${ }^{196}$ (TABLE 3; Supplementary Table 3). TOFA is intracellularly converted into an ester, TOFyl-CoA, which acts as an ACC inhibitor but also reduces cholesterol synthesis ${ }^{197}$, suggesting that it may inhibit ACLY. Given limited bioavailability and lack of specificity, neither agent was commercially developed.

Pfizer identified a series of isozyme-nonspecific ACC inhibitors through HTS which, following a series of optimizations, led to CP-640186 (REF. ${ }^{198}$ ). The crystal structure of the yeast CT domain of ACC in complex with CP-640186 indicates that the compound has tight associations with the active site of the enzyme, blocking biotin binding to the CT domain ${ }^{199}$. Taisho Pharmaceutical ${ }^{200}$ and Takeda Pharmaceuticals ${ }^{201}$ subsequently developed derivatives of CP-640186; however, these compounds had relatively poor metabolic stability and, while subsequent reductions in lipophilicity led to more favourable pharmacokinetics ${ }^{202}$, and the introduction of a 7-methoxy group led to greater potency and metabolic stability ${ }^{203}$, none of these compounds entered clinical testing. Also binding to the CT domain is WZ66, a compound identified via structure-based drug design studies by China Pharmaceutical University, which

\section{Table 3 | ACC inhibitors}

\begin{tabular}{|c|c|c|c|}
\hline Compound (developer) & $\begin{array}{l}\text { Potency in biochemical } \\
\text { assays }\end{array}$ & $\begin{array}{l}\text { Indication and/or preclinical } \\
\text { effects }\end{array}$ & $\begin{array}{l}\text { Clinical trial ID or } \\
\text { refs }\end{array}$ \\
\hline \multicolumn{4}{|l|}{ Clinical stage inhibitors } \\
\hline $\begin{array}{l}\text { Firsocostat (Nimbus } \\
\text { Therapeutics, USA) }\end{array}$ & $\begin{array}{l}\mathrm{IC}_{50}: 2.1 \mathrm{nM}(\mathrm{hACC} 1) \\
6.1 \mathrm{nM}(\mathrm{hACC} 2)\end{array}$ & NASH & $\begin{array}{l}\text { NCT02856555 } \\
\text { (completed, phase II) }\end{array}$ \\
\hline $\begin{array}{l}\text { PF-05221304 (Pfizer Inc., } \\
\text { USA) }\end{array}$ & $\begin{array}{l}\text { IC } \\
90: 13 \mathrm{nM}(\mathrm{hACC} 1) \\
9 \mathrm{nM} \text { (hACC2) }\end{array}$ & NAFLD-NASH & $\begin{array}{l}\text { NCT03248882 } \\
\text { (completed, phase II) }\end{array}$ \\
\hline \multirow[t]{2}{*}{$\begin{array}{l}\text { PF-05175157 (Pfizer Inc., } \\
\text { USA) }\end{array}$} & \multirow[t]{2}{*}{$\begin{array}{l}\mathrm{IC}_{50}: 27 \mathrm{nM}(\mathrm{hACC} 1) \\
33 \mathrm{nM}(\mathrm{hACC} 2)\end{array}$} & Type 2 diabetes & $\begin{array}{l}\text { NCT01792635 } \\
\text { (terminated, phase II) }\end{array}$ \\
\hline & & Acne vulgaris & $\begin{array}{l}\text { NCT02100527 } \\
\text { (withdrawn) }\end{array}$ \\
\hline MK-4074 (Merck \&Co., USA) & $\begin{array}{l}\mathrm{IC}_{50}: \sim 3 \mathrm{nM} \text { (hACC1), } \\
\sim 3 \mathrm{nM}(\mathrm{hACC} 2)\end{array}$ & NAFLD & $\begin{array}{l}\text { NCT01431521 } \\
\text { (completed, phase I) }\end{array}$ \\
\hline \multicolumn{4}{|l|}{ Preclinical stage inhibitors } \\
\hline $\begin{array}{l}\text { A-908292 (Abbott } \\
\text { Laboratories, USA) }\end{array}$ & $\begin{array}{l}\mathrm{IC}_{50}:>30 \mu \mathrm{M}(\mathrm{hACC} 1) \\
0.023 \mu \mathrm{M}(\mathrm{hACC} 2)\end{array}$ & $\begin{array}{l}\text { Reduced plasma lipids } \\
\text { and glycaemia }\end{array}$ & 226 \\
\hline $\begin{array}{l}\text { Carboxamide derivative-1k } \\
\text { (Takeda, Japan) }\end{array}$ & $\begin{array}{l}\text { IC } C_{50}: 170 \mathrm{nM}(\mathrm{hACC} 1) \\
2 \mu \mathrm{M}(\mathrm{hACC} 2)\end{array}$ & $\begin{array}{l}\text { Decreased malonyl-CoA } \\
\text { in xenograft tumour }\end{array}$ & 209 \\
\hline CP-640186 (Pfizer Inc., USA) & $\begin{array}{l}\mathrm{IC}_{55}: 53 \mathrm{nM}(\mathrm{rACC} 1) \\
61 \mathrm{nM}(\mathrm{rACC} 2)\end{array}$ & $\begin{array}{l}\text { Reduced weight gain, hepatic } \\
\text { steatosis, plasma lipids and } \\
\text { glycaemia, and inhibited cancer } \\
\text { growth }\end{array}$ & 216,230 \\
\hline $\begin{array}{l}\text { Monocyclic derivate-1q } \\
\text { (Takeda, Japan) }\end{array}$ & $\begin{array}{l}\mathrm{IC}_{50}: 0.58 \mathrm{nM}(\mathrm{hACC} 1) \\
>10 \mu \mathrm{M}(\mathrm{hACC} 2)\end{array}$ & $\begin{array}{l}\text { Decreased malonyl-CoA in } \\
\text { xenograft tumour }\end{array}$ & 208 \\
\hline $\begin{array}{l}\text { ND-654 (Nimbus } \\
\text { Therapeutics, USA) }\end{array}$ & $\begin{array}{l}\mathrm{IC}_{50}: 3 \mathrm{nM}(\mathrm{hACC} 1) \\
8 \mathrm{nM}(\mathrm{hACC} 2)\end{array}$ & $\begin{array}{l}\text { Inhibited hepatocellular } \\
\text { carcinoma growth, reduced } \\
\text { hepatic steatosis and plasma } \\
\text { lipids }\end{array}$ & 189 \\
\hline $\begin{array}{l}\text { ND-646 (Nimbus } \\
\text { Therapeutics, USA) }\end{array}$ & $\begin{array}{l}\mathrm{IC}_{50}: 3.5 \mathrm{nM}(\mathrm{hACC} 1) \\
4.1 \mathrm{nM}(\mathrm{hACC} 2)\end{array}$ & Inhibited tumour growth & 207 \\
\hline $\begin{array}{l}\text { Olefin derivate-2e (Shionogi } \\
\text { \& Co., Japan) }\end{array}$ & $\begin{array}{l}\mathrm{IC} \mathrm{C}_{50}: 1,950 \mathrm{nM}(\mathrm{hACC} 1) \\
1.9 \mathrm{nM}(\mathrm{hACC} 2)\end{array}$ & Improved glucose homeostasis & 213 \\
\hline $\begin{array}{l}\text { (S)-9c (Boehringer Ingelheim } \\
\text { Pharma GmbH \& Co., USA) }\end{array}$ & $\begin{array}{l}\mathrm{IC}_{50}:>30 \mu \mathrm{M}(\mathrm{hACC} 1) \\
0.07 \mu \mathrm{M}(\mathrm{hACC} 2)\end{array}$ & $\begin{array}{l}\text { Improved glucose and lipid } \\
\text { homeostasis }\end{array}$ & 212 \\
\hline Soraphen A & $K_{\mathrm{i}}: 2.1 \mathrm{nM}(\mathrm{yACC})$ & $\begin{array}{l}\text { Reduced weight gain, improved } \\
\text { insulin sensitivity and inhibited } \\
\text { cancer cell growth }\end{array}$ & 214,228 \\
\hline TOFA & $\mathrm{IC}_{50}: 2.5 \mu \mathrm{M}(\mathrm{rACC})$ & $\begin{array}{l}\text { Reduced lipid synthesis, } \\
\text { inflammation and cancer cell } \\
\text { growth }\end{array}$ & 229 \\
\hline $\begin{array}{l}\text { WZ66 (China Pharmaceutical } \\
\text { University, China) }\end{array}$ & $\begin{array}{l}\mathrm{IC}_{50}: 435.9 \mathrm{nM}(\mathrm{hACC} 1) \\
141.3 \mathrm{nM}(\mathrm{hACC} 2)\end{array}$ & $\begin{array}{l}\text { Reduced hepatic steatosis and } \\
\text { hepatic stellate cell activation }\end{array}$ & 204 \\
\hline
\end{tabular}

ACC, acetyl-CoA carboxylase; hACC, human ACC; $I C_{50}$, half-maximal inhibitory concentration; $K_{d}$, dissociation constant; $K_{i}$, inhibition constant; NAFLD, nonalcoholic fatty liver disease; NASH, nonalcoholic steatohepatitis; rACC, rat ACC; yACC, yeast ACC. 
inhibits recombinant human ACC1 and ACC2 in cells and in rodents ${ }^{204}$

Several pan-ACC inhibitors have recently entered clinical testing in humans. Studies by Merck revealed MK-4074 as a liver-selective ACC inhibitor that dose-dependently inhibited DNL and lowered liver lipids in both rodent and humans ${ }^{48}$ (TABLE 3; Supplementary Table 3). Although the exact binding site of the compound has not been published, it does not appear that clinical development is continuing, potentially owing to the observed induction of hypertriglyceridaemia ${ }^{48}$. Following on from studies with CP-640186, Pfizer developed PF-05221304, as a selective, orally bioavailable and reversible ACC inhibitor that is preferentially distributed to the liver ${ }^{205}$, thereby avoiding potential toxicity related to the inhibition of platelet formation ${ }^{18}$ and developmental defects $^{17}$. Phase II clinical studies with PF-05221304 alone or in combination with a DGAT inhibitor, PF-06865571, in NAFLD-NASH have been completed ${ }^{72}$.

In contrast to PF-05221304, which binds to the CT domain, Nimbus Therapeutics developed several potent ACC inhibitors by focusing on compounds that bind to the $\mathrm{BC}$ domain, blocking dimerization. Briefly, using crystal structure of the human ACC2 BC domain in complex with soraphen $\mathrm{A}$ and subsequent optimization of noncovalent interactions with the dimerization site, they developed a reversible ACC1 and ACC2 inhibitor, ND-630 (REF. ${ }^{206}$ ). In contrast to previous ACC inhibitors, this compound disrupted subunit dimerization by mimicking the effects of AMPK phosphorylation ${ }^{206}$. A second compound of this series, ND-646, exhibited similar potency and mode of inhibition to ND-630, but was developed to be more broadly distributed among peripheral tissues ${ }^{207}$. The third compound, ND-654, was modified to allow for enhanced hepatic uptake ${ }^{189}$. ND-630, also known as GS-0976 (Firsocostat), entered clinical development and is currently in phase II clinical trials for NASH.

In addition to the pan-ACC inhibitors described above, isozyme-specific ACC inhibitors have been developed. Takeda identified novel monocyclic derivatives ${ }^{208}$ and carboxamide derivatives ${ }^{209}$ that demonstrated specificity for ACC1 over ACC2. Abbott Laboratories developed a series of ACC2-specific inhibitors, one of the most potent and selective being A-908292, which exhibited more than 1,000-fold selectivity against ACC2 compared with ACC1. This compound dose-dependently lowered malonyl-CoA in muscle but not liver of rodents ${ }^{210}$. However, a preliminary safety assessment showed neurological and cardiovascular side effects that were resolved by replacement of the alkyne moiety with a heteroaryl linker ${ }^{211}$. Building on this series, Boehringer Ingelheim Pharma and Shionogi research laboratory also identified a series of new molecules that selectively inhibited ACC2 compared with ACC1 without toxicity ${ }^{212,213}$. To the best of our knowledge, none of these agents has entered clinical testing.

$\mathrm{HbA}_{1 \mathrm{c}}$

Haemoglobin $\mathrm{A}_{1 c}\left(\mathrm{HbA}_{1}\right)$ is

a glycated haemoglobin $-\mathrm{a}$ measurement used for the assessment of glycaemic control on-target liabilities. In rodents, soraphen A lowered steatosis and this was associated with improved insulin sensitivity ${ }^{214}$ (Supplementary Fig. 1). However, these improvements in insulin sensitivity did not translate to reductions in blood glucose, as consistent with studies in ACC null mice, ACC inhibition reduced insulin secretion from pancreatic $\beta$-cells ${ }^{215}$. To avoid this problem, TOFA derivatives such as WZ66 were developed that have a preferential liver distribution, reducing hepatic steatosis and hepatic stellate cell activation in mice with diet-induced obesity ${ }^{204}$. CP-640186 also lowers hepatic steatosis and insulin resistance in mice fed a HFD; however, this may be secondary to reductions in adiposity ${ }^{216}$. Lastly, short-term treatment of $d b / d b$ mice with a selective ACC2 inhibitor, $(S)-9 c$, reduced muscle malonylCoA levels and intramyocellular lipids while long-term treatment increased muscle glucose uptake and glucose tolerance while reducing $\mathrm{HbA}_{1 \mathrm{c}}$, postprandial glucose and plasma triacylglycerol levels ${ }^{212}$. These studies suggest that inhibition of ACC is associated with reductions in liver steatosis and modest improvements in glycaemic control.

Given the promising effect of studies evaluating the effects of genetic and pharmacological inhibition of ACC on liver steatosis, several new-generation ACC inhibitors have advanced to clinical trials in NAFLDNASH. In a phase I clinical study, PF-05175157 reduced DNL and increased whole-body fatty acid utilization $^{217}$. However, subsequent clinical trials were terminated owing to extra-hepatic activity leading to reduced platelet count ${ }^{18,205}$. The liver-optimized inhibitor, PF-05221304, was subsequently shown to inhibit DNL, stimulate fatty acid oxidation and reduce triglyceride accumulation in primary human hepatocytes, along with reducing DNL, hepatic steatosis, fibrosis and immune cell activation in preclinical models ${ }^{218}$. Preclinical studies designed to assess the level of liver targeting achieved with PF-05221304 showed a maximal reduction of $82 \%$ in hepatic DNL compared with up to $33 \%$ reductions in lung and bone marrow ${ }^{219}$. In phase I clinical trials, PF-05221304 inhibited hepatic DNL in a dose-dependent manner without affecting platelet count, consistent with its more liver-targeted actions ${ }^{205}$. And in phase II clinical trials for NAFLD, PF-05221304 dose-dependently reduced liver fat by up to $65 \%$ at the highest dose while also lowering $\mathrm{HbA}_{1 \mathrm{c}}\left(\mathrm{REF}^{72}\right)$.

Studies using the liver-specific inhibitor MK-4074 in rodents and humans have supported the therapeutic potential of potent ACC inhibition, but they have also raised additional questions about on-target liabilities. In a phase I clinical trial, MK-4074 reduced hepatic steatosis by $36 \%$ after 4 weeks of treatment ${ }^{48}$. In a preclinical model of NASH, the compound decreased liver fibrosis by $19 \%$ in addition to reducing liver fat ${ }^{220}$. However, clinical development of MK-4074 has been discontinued.

ND-630 is preferentially distributed to liver ${ }^{206}$ and shows favourable effects in both preclinical and clinical studies. ND-630 dose-dependently reduces hepatic steatosis and hyperinsulinaemia in rodents ${ }^{206}$. An independent group has confirmed these effects on hepatic steatosis in animals fed a Western diet and also observed reductions in fibrosis ${ }^{221}$. In line with these studies, 
ND-630 inhibits hepatic stellate cell activation to reduce fibrogenic activity ${ }^{222}$. In a phase I clinical study, ND-630/ GS-0976 was well tolerated and inhibited hepatic DNL in subjects with obesity and/or overweight ${ }^{223}$. In an open labelled, non-placebo-controlled study, patients with NASH treated with ND-630 had a median decrease of $22 \%$ in hepatic DNL and a significant reduction in hepatic fat and liver stiffness after 12 weeks of treatment ${ }^{4}$. In a subsequent larger randomized, placebo-controlled study, ND-630 treatment reduced hepatic steatosis by $21 \%{ }^{224}$. ND-630 has recently been tested in combination with the apoptosis signalregulating kinase 1 (ASK1) inhibitor selonsertib and farnesoid X receptor (FXR) agonist cilofexor in patients with bridging fibrosis or compensated cirrhosis, with combinations showing greater improvement in NASH activity than ND-630 alone ${ }^{225}$. Phase II studies with ND-630 and the GLP1R-agonist semaglutide are currently in progress.

Cardiovascular disease. Circulating triglycerides are an important risk factor for CVD, and in preclinical studies in mice, several ACC inhibitors, such as CP-640186 (REF. ${ }^{216}$ ), A-908292 (REF. ${ }^{226}$ ), (S)-9c ${ }^{212}$, ND-630 (REF. ${ }^{206}$ ) and ND-654 (REF ${ }^{189}$ ) lowered plasma triglyceride levels. However, in humans, ACC inhibitors dose-dependently increase plasma triglycerides and/or VLDL. As originally described for MK-4074 (REF. ${ }^{48}$ ), but also observed with $\mathrm{PF}-05221304\left(\mathrm{REF}^{72}\right)$, this increase in circulating triglycerides is likely mediated by the effects of decreased liver polyunsaturated fatty acids to stimulate SREBP1c activation, leading to increased GPAT and VLDL secretion ${ }^{48}$. ND-630 also increases plasma triglycerides ${ }^{224}$, an effect associated with reduced triglyceride clearance ${ }^{227}$. To avoid this adverse effect, preclinical studies have been undertaken that combine ACC inhibitors with agents that reduce plasma triglycerides such as omega-3 fatty acids $^{48}$ and PPAR $\alpha$ agonists such as fenofibrate ${ }^{227}$. Similarly, DGAT2 inhibition also blocked the effects of PF-05221304, to increase liver SREBP1c and circulating triglycerides while exerting additive effects on liver fibrosis in rodent models ${ }^{72}$. Importantly, in phase II clinical trials, DGAT2 inhibition also mitigated PF-05221304-induced increases in serum triglycerides and ApoaC3 (REF. ${ }^{72}$ ). These data suggest that combination strategies may be an effective means to improve the cardiovascular risk profile of ACC inhibitors in patients with NASH.

Cancer. Studies of early ACC inhibitors, soraphen $\mathrm{A}^{228}$ and TOFA ${ }^{229}$ demonstrated inhibitory effects on multiple cancer types. Although the contribution of ACC inhibition to the effects of these early inhibitors is unknown, the anti-tumorigenesic effect of ACC inhibition has been supported by more recent studies using inhibitors with improved potency and specificity. For example, CP-640186 reduced the growth of human lung cancer cells $^{230}$, while ND-646 and ND-654 reduced tumour burden in mouse models of non-small-cell lung cancer ${ }^{207}$ and hepatocellular carcinoma ${ }^{189}$, respectively, when used alone and in combination with existing standards of care.

\section{FAS}

\section{Regulation and structure}

Human FAS is highly expressed in adipose tissues and reproductive organs (see Related links). In contrast to ACLY and ACC, few allosteric and covalent mechanisms regulating the activity of FAS have been described (FIC. 3). In yeast, phosphorylation of Ser1140, Ser 1640 and Ser1827 are associated with increased 18:0-CoA production $^{231}$, while in a breast cancer cell line, FAS is phosphorylated at Tyr66 when in complex with human epidermal growth factor receptor 2 (REF. ${ }^{232}$ ). In addition, FAS is degraded by E3 ubiquitin ligase COP1 in the presence of adaptor protein SHP2 and deubiquitinated by ubiquitin-specific protease $2 \mathrm{a}^{233}$ (FIG. 3). These data suggest that under some conditions and cell types, FAS may be regulated by covalent and ubiquitylation mechanisms; however, it appears that the regulation of FAS primarily occurs through transcriptional control.

FAS has evolved structurally and functionally different variants that are generally divided into two types based on the organization of their catalytic units - type 1 and type $2 \mathrm{FAS}^{234,235}$. In eukaryotes, the multifunctional complex of type 1 FAS is expressed in the cytosol and regulated by only one gene ${ }^{234}$. Type 2 FAS is present in mitochondria and functions completely independently of the cytosolic type 1 FAS $^{235}$. Type 1 FAS typically generates only palmitate, whereas type 2 FAS is able to produce diverse fatty acids, including unsaturated fatty $\operatorname{acids}^{236}$. Structurally, mammalian FAS is a homodimer protein that consists of two multifunctional polypeptides, containing seven functional domains and two non-enzymatic or pseudo domains in each chain ${ }^{237}$, all of which are required for fatty acid biosynthesis (FIG. 4).

\section{Pharmacological inhibitors}

Several natural products and their derivatives have been found to inhibit FAS. Cerulenin, an antifungal antibiotic originally isolated from Cephalosporium caerulens inhibits FAS in both mammals and bacteria ${ }^{238}$. And although cerulenin inhibits FAS by binding covalently to the active cysteine thiol in the $\beta$-ketoacyl synthase (KS) domain (FIG. 4), it also inhibits HMG-CoA synthetase activity $^{238}$, blocking sterol synthesis. The reactivity and lack of specificity of cerulenin led to the development of the synthetic analogue $\mathrm{C75}$, which binds to the $\mathrm{KS}^{239}$ and thioesterase (TE) domains of human FAS ${ }^{240}$.

Several novel small-molecule FAS inhibitors have been developed that inhibit the TE domain. Orlistat, also known as tetrahydrolipstatin, is a derivative of lipstatin, a naturally occurring inhibitor of pancreatic lipase, and was found to also inhibit FAS through irreversible binding to the TE domain of the enzyme ${ }^{241}$. Owing to poor oral bioavailability and metabolic instability, several reformulation efforts were initiated, including a hydrophilic nanoparticle delivery system ${ }^{242}$, poly(ethylene glycol)-conjugated poly(lactic-co-glycolic acid) nanoparticles ${ }^{243}$ and folate receptor-targeted micellar nanoparticles ${ }^{244}$; however, none of these has moved into clinical development. Fasnall, a thiophenopyrimidine derivative also targets cofactor binding sites in multiple domains and in pharmacokinetic and toxicity studies shows no toxic effects ${ }^{245}$. More recently, triazole 
Table 4 | FAS inhibitors

\begin{tabular}{|c|c|c|c|}
\hline Compound (developer) & $\begin{array}{l}\text { Potency in } \\
\text { biochemical assays }\end{array}$ & $\begin{array}{l}\text { Indication and/or } \\
\text { preclinical effects }\end{array}$ & Clinical trial ID or refs \\
\hline \multicolumn{4}{|l|}{ Clinical stage inhibitors } \\
\hline Orlistat & $\mathrm{IC}_{50}: 100 \mathrm{nM}$ & Obesity & Approved \\
\hline \multirow[t]{2}{*}{$\begin{array}{l}\text { TVB-2640 (Sagimet Biosciences, } \\
\text { USA) }\end{array}$} & \multirow[t]{2}{*}{$\mathrm{IC}_{50}<0.05 \mu \mathrm{M}$} & $\mathrm{NASH}$ & $\begin{array}{l}\text { NCT04906421 } \\
\text { (in progress, phase II) }\end{array}$ \\
\hline & & $\begin{array}{l}\text { Lung carcinoma, breast cancer, } \\
\text { astrocytoma, colon cancer }\end{array}$ & $\begin{array}{l}\text { NCT03808558, } \\
\text { NCT03179904, } \\
\text { NCT03032484, } \\
\text { NCT02980029 (in } \\
\text { progress, phase I/II) }\end{array}$ \\
\hline $\begin{array}{l}\text { FT-4101 (Forma Therapeutics, } \\
\text { USA) }\end{array}$ & $\mathrm{IC}_{50}: 40 \mathrm{nM}$ & NASH & $\begin{array}{l}\text { NCT04004325 } \\
\text { (terminated, phase I/II) }\end{array}$ \\
\hline \multicolumn{4}{|l|}{ Preclinical stage inhibitors } \\
\hline $\begin{array}{l}\text { BI-99179 (Boehringer Ingelheim } \\
\text { Pharma GmbH \& Co, Germany) }\end{array}$ & $\mathrm{IC}_{50}: 79 \mathrm{nM}$ & Inhibited cancer cell growth & 249 \\
\hline Cerulenin & $I C_{50}: 4.5 \mu \mathrm{M}$ & $\begin{array}{l}\text { Reduced weight gain and } \\
\text { inhibited cancer cell growth }\end{array}$ & 253,261 \\
\hline $\begin{array}{l}\text { C75 (Johns Hopkins Medical } \\
\text { Institutions, USA) }\end{array}$ & $\mathrm{IC}_{50}: 15.5 \mu \mathrm{M}$ & $\begin{array}{l}\text { Reduced body weight, hepatic } \\
\text { steatosis and blood glucose, } \\
\text { and inhibited cancer growth }\end{array}$ & 255,257 \\
\hline $\begin{array}{l}\text { Fasnall (Duke University School } \\
\text { of Medicine, USA) }\end{array}$ & $\mathrm{IC}_{50}: 3.71 \mu \mathrm{M}$ & Inhibited cancer cell growth & 245 \\
\hline $\begin{array}{l}\text { GSK2194069 (GlaxoSmithKline, } \\
\text { USA) }\end{array}$ & $\mathrm{IC}_{50}: 7.7 \mathrm{nM}$ & Inhibited cancer cell growth & 246 \\
\hline $\begin{array}{l}\text { IPI-9119 (Dana-Farber Cancer } \\
\text { Institute, USA) }\end{array}$ & $I C_{50}: 0.3 \mathrm{nM}$ & Inhibited cancer cell growth & 247 \\
\hline $\begin{array}{l}\text { MP-ML-24-N1 (University } \\
\text { Hospital Tübingen, Germany) }\end{array}$ & $\mathrm{IC}_{50}: 1.6 \mu \mathrm{M}$ & Inhibited cancer cell growth & 246 \\
\hline $\begin{array}{l}\text { TVB-3166 (Sagimet Biosciences, } \\
\text { USA) }\end{array}$ & $\mathrm{IC}_{50}: 42 \mathrm{nM}$ & Inhibited cancer cell growth & 250 \\
\hline
\end{tabular}

FAS, fatty acid synthase; $\mathrm{IC}_{50}$, half-maximal inhibitory concentration; $\mathrm{NASH}$, nonalcoholic steatohepatitis.

urea-based substitutions to Fasnall, led to the identification of MP-ML-24-N1, which inhibits FAS through the TE domain and has good cellular permeability ${ }^{246}$. Lastly, IPI-9119 irreversibly inhibits the TE domain by promoting acetylation of the catalytic serine residue and has shown high potency and selectivity against FAS as well as good pharmacological properties ${ }^{247}$.

FAS inhibitors that target the $\beta$-ketoacyl reductase (KR) domain have also been developed with some recently entering clinical testing. GSK identified GSK2194069 using an HTS approach based on compound competition with NADPH for binding in the KR domain ${ }^{248}$. Inhibitor-bound X-ray crystal structures showed that the compound forms hydrogen bonds with residues Ser2021 and Thyr2034, while studies in various cell lines indicated good cellular activity towards inhibiting $\mathrm{DNL}^{248}$. Using a similar approach, Boehringer Ingelheim Pharma GmbH \& Co identified BI-99179 as a potent and selective inhibitor for human FAS that is also assumed to bind to the KR domain ${ }^{249}$. BI-99179 showed high metabolic stability in rat and human microsomes and high oral bioavailability in rats $^{249}$. Lastly, Sagimet Biosciences developed TVB-3166, an orally bioavailable, reversible, potent and selective inhibitor of FAS that also likely inhibits the KR domain ${ }^{250}$. A related but more advanced compound, TVB-2640, is under clinical development. Recently, Forma Therapeutics reported that FT-4101 inhibited human FAS by also targeting the KR domain ${ }^{251}$ (TABLE 4; Supplementary Table 4).

\section{Therapeutic indications}

Obesity. The link between FAS inhibition and reductions in body weight is not well understood. Initial observations that cerulenin induced weight loss in both lean ${ }^{252}$ and obese ${ }^{253}$ mice suggested that effects may be primarily related to toxicity rather than inhibition of FAS. However, subsequent studies showed that C75 also had a profound effect on body weight ${ }^{254,255}$ (Supplementary Fig. 1), suggested that effects may involve an increase in energy expenditure and suppression of appetite. Although the FAS inhibitor orlistat has been approved for weight management in obesity it is important to note that owing to poor bioavailability the inhibition of pancreatic lipase, not FAS, is the primary mechanism by which this agent induces weight loss. Importantly, data from selective FAS inhibitors such as TVB-2640 do not support a link with weight loss.

NAFLD-NASH and type 2 diabetes. Early studies demonstrated that treatment with $\mathrm{C} 75$ decreases hepatic steatosis ${ }^{256}$ and blood glucose in mouse models of obesity $^{252,257}$. These findings supported the development 
of TVB-2640, which was found to lower DNL by up to $90 \%$ in individuals with obesity and insulin resistance ${ }^{258}$. Phase II clinical studies are currently underway in patients with NASH. FT-4101 has been tested in humans with obesity, where it suppressed DNL and lowered steatosis ${ }^{251}$. However, clinical development has ceased for reasons that have not been disclosed. Although these studies support a potential therapeutic benefit of inhibiting FAS in $\mathrm{NASH}$, results from the ongoing phase II studies will be imperative to better understand safety and efficacy.

Cancer. Although cerulenin has beneficial effects in various cancer cell lines and xenograft tumour models ${ }^{259-261}$, reactivity and the profound weight loss caused by this compound limited its potential. Antitumour effects of C75 were also demonstrated in various cancer cell lines and xenograft tumours alone $e^{261}$ and in combination with radiotherapy ${ }^{93}$. In addition, orlistat exhibits cytostatic and cytotoxic effects in tumour cells, and nanoparticle technology has been used to improve bioavailability and subsequent cytotoxicity in xenografts ${ }^{241,243,244}$. Studies have been conducted with more-specific inhibitors that strengthen FAS as an anticancer target. Fasnall was shown to inhibit breast cancer ${ }^{245}$ and prostate cancer cell growth $^{262}$, and GSK2194069 inhibits the proliferation of non-small-cell lung cancer cell lines ${ }^{248}$. IPI-9119 $\left(\right.$ REF. $\left.^{247}\right)$ and TVB-3166 $\left(\mathrm{REF}^{250}\right)$ also inhibit cancer cell proliferation in several distinct preclinical models. Interestingly, recent studies have found that owing to limited exogenous fatty acids within the brain, metastasis of breast cancer is highly sensitive to FAS inhibition by TVB-3166 and BI-99179 (REF. ${ }^{263}$ ). Phase I clinical trials with TVB2640 have been completed in healthy subjects and in patients with solid malignant tumours ${ }^{264}$, and phase II studies in multiple cancers are underway. Results from these studies will be important to better understand the potential therapeutic effects of FAS inhibition in cancer.

\section{Challenges}

In the past 50 years tremendous progress has been made in understanding the biochemical mechanisms and physiological significance of DNL in regulating cellular metabolism and whole-body energy homeostasis. Important steps along the way have included biochemical identification of the key metabolic intermediates in the conversion of glucose to fatty acids, the molecular cloning of the key enzymes regulating the process and the discovery of crucial allosteric and covalent mechanisms that regulate flux through the pathway. Subsequent studies in genetically modified mice revealed the physiological role of DNL, broadening our understanding of the complex connections that exist between multiple cellular processes and tissues well beyond the simple storage of excess calories in adipose tissue. Meanwhile, advances in structural biology laid the foundation for the molecular underpinnings by which natural products and new-generation small molecules inhibited enzyme activity. Collectively, this information led to an explosion in the number of novel small molecules that have been developed and tested in preclinical models for cardiometabolic disease, cancer and other indications. Although these compounds vary in their molecular target, chemical structure and physicochemical properties, the common action in which DNL is inhibited supports the notion that it is beneficial for the prevention and treatment of a broad spectrum of diseases. However, the development and testing of this new generation of highly selective and efficacious small molecules has also revealed several surprising new biological insights that have important implications for safety, efficacy and the need for combination therapies as they progress into clinical trials.

\section{Safety-related side effects}

There are several safety concerns associated with inhibiting DNL. The first and most significant to be identified was related to embryonic development. Mice homozygous deficient for ACLY ${ }^{265}$, ACC1 $\left(\mathrm{REF}^{266}\right)$ or $\mathrm{FAS}^{267}$ die during embryonic development, and CIC deficiency is characterized by severe neurodevelopmental disorders ${ }^{268}$. The ACC inhibitor PF-05175157 also induces developmental toxicity (growth retardation and dysmorphogenesis associated with disrupted midline fusion) in rats and rabbits ${ }^{17}$. Thus, avoiding systemic inhibition of DNL is essential to safeguard embryonic development.

Another major concern with inhibiting DNL in lipogenic organs such as liver and adipose tissue is that this simply redirects carbon into other parts of the body where toxicity may be even greater. Experimental evidence of this effect was first established with the ACC inhibitor MK-4074, which dose-dependently increased SREBP1c and serum triglycerides; an effect that was shown to be 'on-target' as mice genetically lacking ACC in the liver had a similar phenotype ${ }^{48}$. Subsequent studies with ND-630 (REF. ${ }^{224}$ ) and PF-05221304 (REF. ${ }^{205}$ ) in humans with NASH revealed similar effects. Importantly, increases in serum triglycerides by PF- 05221304 can be inhibited by co-administration of a DGAT2 inhibitor ${ }^{72}$. Interestingly, inhibiting ACC by activating AMPK ${ }^{269,270}$ lowers serum triglycerides and cholesterol effects. Similar results are also observed with the inhibition of ACLY ${ }^{173,181,182}$. A greater understanding of the mechanism behind these differential responses to inhibiting liver DNL will be important for alleviating potential adverse events.

The upstream and downstream consequences associated with blockade at different steps in the DNL pathway, leading to the accumulation of metabolic intermediates, must also be considered. For example, inhibition of FAS leads to a build-up of malonyl-CoA. This increase in malonyl-CoA as a result of FAS inhibition impairs physiological and pathological angiogenesis via the malonylation of $\mathrm{mTOR}^{271}$. Malonylation has also been implicated in various metabolic pathways ${ }^{272}$, histone modifications $^{273}$ and immune cell reprogramming ${ }^{274}$, suggesting that FAS inhibition may exhibit broader biological effects than the anticipated DNL inhibition. CIC inhibition may also pose safety concerns, as a deficiency of CIC increases mitochondrial citrate and isocitrate and this is associated with severe neurometabolic effects ${ }^{268}$. Lastly, ACLY inhibition, which lowers acetyl-CoA, may affect the acetylation profile of many different histones, potentially having a wide array of differential effects on gene expression profiles and epigenetic programming ${ }^{275}$. 
Thus, it is pivotal to consider the effects of metabolic intermediates when developing DNL inhibitors as a therapeutic approach.

\section{Bypassing DNL inhibition/compensatory pathways}

The concept of cellular metabolic flexibility is another key concern for DNL inhibitors. Metabolism is fluid and interconnected: a block in one pathway enhances flux through alternative pathways. This has been studied most intensely in cancer in which plasticity is a hallmark of chemoresistance. For example, in response to reduced flux from glucose to acetyl-CoA under hypoxic conditions, mutations in KRAS have been shown to provide cancer cells with increased ability to scavenge exogenous lipids, thereby diminishing dependence on DNL ${ }^{276}$. The reciprocal relationship is also observed whereby inhibiting exogenous fatty acid uptake by blocking CD36 enhances the efficacy of the FAS inhibitor C75 (REF. ${ }^{277}$ ). Similarly, in breast cancer brain metastasis, where exogenous fatty acids are limited, inhibiting FAS exerts greater lethality compared with the liver where exogenous fatty acids are abundant ${ }^{263}$. Inhibition of ACLY can also be bypassed in some cancer cells ${ }^{278}$ and in the liver of mice fed a high-fructose diet ${ }^{45}$ through upregulation of ACSS2. These data suggest that depending on the nutritional context and cell types involved, for DNL inhibitors to be highly effective it may be necessary to also target compensatory mechanisms. For example, might it be important for people being treated with an ACLY inhibitor to limit their alcohol and fructose intake to maximize effects? Alternatively, might an individual having their cancer treated with a FAS inhibitor need to limit fatty foods? Despite these compelling data in preclinical models, data generated in clinical settings with respect to ACLY inhibition for LDL lowering ${ }^{17,181,182}$, ACC inhibitors for NASH ${ }^{48,72,205,224}$ and FAS inhibition for cancer ${ }^{264}$ suggest that the degree of DNL inhibition achieved may be sufficient to overcome any compensatory pathways. However, further studies investigating this will be important.

\section{Systemic/organ-specific inhibition}

A significant challenge with the clinical development of DNL inhibitors has been the discovery of differential responses of certain cells between rodents and humans. An example of this occurred with the clinical development of the systemic ACC inhibitor PF-05175157, which was found to have no toxicity in rodents but dose escalation studies in people revealed reduced platelet count ${ }^{18,205}$. Subsequent studies established that the reduction in platelet count was due to ACC inhibition within bone marrow, which impaired megakaryocyte maturation ${ }^{18}$. This finding demonstrated a requirement for DNL in humans but not rodents, which was especially surprising given that DNL in tissues such as liver and adipose tissue is much higher in rodents than in humans ${ }^{279}$. Importantly, these effects have been avoided with new-generation liver-targeted ACC inhibitors ND-630 (REF. ${ }^{224}$ ) and PF-05221304 (REF. ${ }^{205}$ ). Similarly, genetic inhibition of ACLY in muscle and adipose tissue results in muscle weakness and lipodystrophy, respectively, but has been avoided by the development of the liver-targeted prodrug bempedoic acid ${ }^{167}$. Liver-specific inhibition of DNL may also be helpful to avoid the potential detrimental effect of adipose tissue DNL inhibition on whole-body insulin sensitivity, given that adipose tissue DNL directly correlates with insulin sensitivity ${ }^{61,280}$. Lastly, FAS inhibition is associated with alopecia, an effect that has been reduced with the new generation of liver-directed FAS inhibitors TVB-2640 $\left(\right.$ REF. $\left.^{264}\right)$ and FT-4101 (REF. ${ }^{251}$ ). Thus, data presented to date suggest that inhibition of FAS, ACC and ACLY can be safely achieved through liver targeting; however, whether chronically inhibiting DNL in other organs or cell types can be safely achieved requires further study.

\section{Outlook}

Over the past decade, many challenges have been addressed leading to DNL inhibitors entering clinical development for cancer, CVD, NASH and acne vulgaris. In the era of single-cell analysis of cellular metabolism, an especially exciting avenue of research will be to determine whether there may be specific cell types in which DNL is upregulated under different pathological conditions. The identification of these potential cell types may lead to cell-specific DNL inhibitors that could be applied during certain windows of development for alleviating or potentially preventing disease. Another important consideration will be related to balancing efficacy and safety as enhanced potency may lead to greater compensatory upregulation and/or toxicity. An example of this is seen with the ACC inhibitors whereby inhibition of DNL closely parallels concomitant increases in circulating triglycerides and inhibition of platelets. As DNL is transiently upregulated after a meal, one way to potentially avoid upregulation of compensatory pathways and/or toxicity may involve the use of inhibitors with a shorter half-life so that DNL is maintained at basal levels. Ultimately, whether DNL inhibitors are safe and effective enough to be used as a monotherapy or will be used in combination with other therapies to enhance efficacy or offset liabilities, as has been proposed for ACC inhibitors in NASH, will depend on clinical trial results. In this regard, findings with the ACLY inhibitor bempedoic acid are encouraging as they suggest that chronic inhibition of DNL in the liver is safe when used alone or with other standards of care. Whether DNL inhibitors that target other cell types and organ systems become widely adopted as a cornerstone for treating other disease indications beyond CVD and NASH remains to be determined.

Published online 14 January 2022
1. Smedley, I. \& Lubrzynska, E. The biochemical synthesis of the fatty acids. Biochem. J. 7, 364-374 (1913).

2. Lai, H. T. M. et al. Serial plasma phospholipid fatty acids in the de novo lipogenesis pathway and total mortality, cause-specific mortality, and cardiovascular diseases in the cardiovascular health study. J. Am Heart Assoc. 8 , e012881 (2019). The first prospective study in adults to investigate the associations of DNL-related fatty acid biomarkers with mortality and incident CVD.
3. Ference, B. A. et al. Mendelian randomization study of $A C L Y$ and cardiovascular disease. N. Engl. J. Med. 380, 1033-1042 (2019).

Demonstrates that genetic variants that mimic ACLY inhibitors and statins lower plasma LDL in humans by the same mechanism of action. 
4. Lawitz, E. J. et al. Acetyl-CoA carboxylase inhibitor CS-0976 for 12 weeks reduces hepatic de novo lipogenesis and steatosis in patients with nonalcoholic steatohepatitis. Clin. Gastroenterol. Hepatol. 16, 1983-1991.e3 (2018).

5. Smith, G. I. et al. Insulin resistance drives hepatic de novo lipogenesis in nonalcoholic fatty liver disease. J. Clin. Invest. 130, 1453-1460 (2020).

6. Imamura, F. et al. Fatty acids in the de novo lipogenesis pathway and incidence of type 2 diabetes: a pooled analysis of prospective cohort studies. PLoS Med. 17, e1003102 (2020).

7. Wen, J. et al. ACLY facilitates colon cancer cell metastasis by CTNNB1. J. Exp. Clin. Cancer Res. 38 401 (2019).

8. Chen, Y. et al. mTOR complex-2 stimulates acetyl-CoA and de novo lipogenesis through ATP citrate lyase in HER2/PIK3CA-hyperactive breast cancer. Oncotarget 7, 25224-25240 (2016).

9. Munger, J. et al. Systems-level metabolic flux profiling identifies fatty acid synthesis as a target for antiviral therapy. Nat. Biotechnol. 26, 1179-1186 (2008).

10. Du, Y. et al. mRNA display with library of evendistribution reveals cellular interactors of influenza virus NS1. Nat. Commun. 11, 2449 (2020).

11. Yang, Z., Matteson, E. L., Goronzy, J. J. \& Weyand, C. M. T-cell metabolism in autoimmune disease. Arthritis Res. Ther. 17, 29 (2015)

12. Endo, Y. et al. Obesity drives Th17 cell differentiation by inducing the lipid metabolic kinase, ACC1. Cell Rep. 12, 1042-1055 (2015)

13. Carvajal-Gonzalez, S. et al. Human sebum requires de novo lipogenesis, which is increased in acne vulgaris and suppressed by acetyl-CoA carboxylase inhibition. Sci. Transl. Med. 11, eaau8465 (2019).

14. Knobloch, M. et al. Metabolic control of adult neura stem cell activity by Fasn-dependent lipogenesis. Nature 493, 226-230 (2013)

15. Isaev, N. K., Stelmashook, E. V. \& Genrikhs, E. E. Neurogenesis and brain aging. Rev. Neurosci. 30 573-580 (2019).

16. Markham, A. Bempedoic acid: first approval. Drugs 80, 747-753 (2020)

17. Catlin, N. R. et al. Inhibition of ACC causes malformations in rats and rabbits: comparison of mammalian findings and alternative assays. Toxicol. Sci. 28, 183-194 (2020).

18. Kelly, K. L. et al. De novo lipogenesis is essential for platelet production in humans. Nat. Metab. 2 1163-1178 (2020)

19. Das, S. et al. ATP citrate lyase improves mitochondrial function in skeletal muscle. Cell Metab. 21, 868-876 (2015).

20. Zhao, S. et al. ATP-citrate lyase controls a glucoseto-acetate metabolic switch. Cell Rep. 17, 1037-1052 (2016)

First study to describe the generation of Aclyfloxed mice and ACLY-null mouse embryonic fibroblasts. Using these models of ACLY deficiency, the authors show that this leads to upregulation of ACSS2 and scavenging of acetate to maintain acetyl-CoA.

21. Huang, Z. et al. ACSS2 promotes systemic fat storage and utilization through selective regulation of genes involved in lipid metabolism. Proc. Natl Acad. Sci. USA 115, E9499-E9506 (2018).

22. Liu, X. et al. Acetate production from glucose and coupling to mitochondrial metabolism in mammals. Cell 175, 502-513.e13 (2018).

23. Metallo, C. M. et al. Reductive glutamine metabolism by IDH 1 mediates lipogenesis under hypoxia. Nature 481, 380-384 (2011).

24. Jiang, L. et al. Quantitative metabolic flux analysis reveals an unconventional pathway of fatty acid synthesis in cancer cells deficient for the mitochondrial citrate transport protein. Metab. Eng. 43, 198-207 (2017).

25. Filipp, F. V., Scott, D. A., Ronai, Z. A., Osterman, A. L. $\&$ Smith, J. W. Reverse TCA cycle flux through isocitrate dehydrogenases 1 and 2 is required for lipogenesis in hypoxic melanoma cells. Pigment. Cell Melanoma Res. 25, 375-383 (2012).

26. Bideyan, L., Nagari, R. \& Tontonoz, P. Hepatic transcriptional responses to fasting and feeding. Genes Dev. 35, 635-657 (2021)

27. Galsgaard, K. D., Pedersen, J., Knop, F. K., Holst, J. J. \& Wewer Albrechtsen, N. J. Glucagon receptor signaling and lipid metabolism. Front. Physiol. 10 413 (2019).

28. Ferguson, D. \& Finck, B. N. Emerging therapeutic approaches for the treatment of NAFLD and type 2 diabetes mellitus. Nat. Rev. Endocrinol. 17, 484-495 (2021).

29. Viscarra, J. \& Sul, H. S. Epigenetic regulation of hepatic lipogenesis: role in hepatosteatosis and diabetes. Diabetes 69, 525-531 (2020).

30. Solinas, G., Borén, J. \& Dulloo, A. G. De novo lipogenesis in metabolic homeostasis: more friend than foe? Mol. Metab. 4, 367-377 (2015).

31. Yoon, H., Shaw, J. L., Haigis, M. C. \& Greka, A. Lipid metabolism in sickness and in health: emerging regulators of lipotoxicity. Mol. Cell 81, 3708-3730 (2021).

32. Chakravarthy, M. V. et al. Brain fatty acid synthase activates PPARalpha to maintain energy homeostasis. J. Clin. Invest. 117, 2539-2552 (2007).

33. Santos, G. A. et al. Hypothalamic inhibition of acetyl-CoA carboxylase stimulates hepatic counterregulatory response independent of AMPK activation in rats. PLOS ONE 8, e62669 (2013).

34. Wolfgang, M. J. \& Lane, M. D. The role of hypothalamic malonyl-CoA in energy homeostasis. J. Biol. Chem. 281, 37265-37269 (2006).

35. Galic, S. et al. AMPK signaling to acetyl-CoA carboxylase is required for fasting- and cold-induced appetite but not thermogenesis. eLife 7, e32656 (2018).

36. Virtanen, K. A. et al. Functional brown adipose tissue in healthy adults. N. Engl. J. Med. 360, 1518-1525 (2009).

37. Adlanmerini, M. et al. Circadian lipid synthesis in brown fat maintains murine body temperature during chronic cold. Proc. Natl Acad. Sci. USA 116 18691-18699 (2019).

38. Blondin, D. P. et al. Inhibition of intracellular triglyceride lipolysis suppresses cold-induced brown adipose tissue metabolism and increases shivering in humans. Cell Metab. 25, 438-447 (2017).

39. Sanchez-Gurmaches, J. et al. Brown fat AKT2 is a cold-induced kinase that stimulates ChREBP-mediated de novo lipogenesis to optimize fuel storage and thermogenesis. Cell Metab. 27, 195-209.e6 (2018).

40. Lodhi, I. J. et al. Inhibiting adipose tissue lipogenesis reprograms thermogenesis and PPAR $\gamma$ activation to decrease diet-induced obesity. Cell Metab. 16 189-201 (2012)

41. Yuan, F. et al. Activation of GCN2/ATF4 signals in amygdalar PKC- $\delta$ neurons promotes WAT browning under leucine deprivation. Nat. Commun. 11, 2847 (2020).

42. Higuchi, N. et al. Liver $\mathrm{X}$ receptor in cooperation with SREBP-1 $\mathrm{C}$ is a major lipid synthesis regulator in nonalcoholic fatty liver disease. Hepatol. Res. 38 1122-1129 (2008)

43. Tan, M. et al. Inhibition of the mitochondrial citrate carrier, SIc25a1, reverts steatosis, glucose intolerance and inflammation in preclinical models of NAFLD/NASH. Cell Death Differ. 27, 2143-2157 (2020). First paper showing genetic inhibition of CIC (also known as SLC25A1) in the liver and that pharmacological inhibition of CIC using CTPI-2 reduces hepatic steatosis and glucose intolerance.

44. Wang, Q. et al. Abrogation of hepatic ATP-citrate lyase protects against fatty liver and ameliorates hyperglycemia in leptin receptor-deficient mice. Hepatology 49, 1166-1175 (2009).

45. Zhao, S. et al. Dietary fructose feeds hepatic lipogenesis via microbiota-derived acetate. Nature 579, 586-591 (2020)

46. Mao, J. Liver-specific deletion of acetyl-CoA carboxylase 1 reduces hepatic triglyceride accumulation without affecting glucose homeostasis. Proc. Natl Acad. Sci. USA 103, 8552-8557 (2006)

47. Savage, D. B. et al. Reversal of diet-induced hepatic steatosis and hepatic insulin resistance by antisense oligonucleotide inhibitors of acetyl-CoA carboxylases 1 and 2. J. Clin. Invest. 116, 817-824 (2006). First study showing that genetic inhibition of both ACC1 and ACC2 in the liver reduces hepatic steatosis and improves insulin sensitivity.

48. Kim, C. W. et al. Acetyl CoA carboxylase inhibition reduces hepatic steatosis but elevates plasma triglycerides in mice and humans: a bedside to bench investigation. Cell Metab. 26, 394-406 (2017). Demonstrates that ACC inhibitors reduce hepatic steatosis but also increase plasma triglycerides in humans. Subsequent studies in ACC hepatocytespecific null mice show that this is an 'on-target' effect associated with activation of SREBP1 $c$ and increases in GPAT, which can be inhibited with fish oil.

49. Fullerton, M. D. et al. Single phosphorylation sites in Acc1 and Acc2 regulate lipid homeostasis and the insulin-sensitizing effects of metformin. Nat. Med. 19, 1649-1654 (2013)

Demonstrates additivity between ACC1 and ACC2 in the liver towards regulating liver DNL and fatty acid oxidation and that AMPK phosphorylation and inhibition of ACC1 and ACC2 reduces liver steatosis, fibrosis and insulin resistance.

50. Chow, J. D. et al. Genetic inhibition of hepatic acetylCoA carboxylase activity increases liver fat and alters global protein acetylation. Mol. Metab. 3, 419-431 (2014).

51. Chakravarthy, M. V. et al. "New" hepatic fat activates PPARalpha to maintain glucose, lipid, and cholesterol homeostasis. Cell Metab. 1, 309-322 (2005)

52. Roumans, K. H. M. et al. Hepatic saturated fatty acid fraction is associated with de novo lipogenesis and hepatic insulin resistance. Nat. Commun. 11, 1891 (2020).

53. Takagi, H. et al. ACC2 deletion enhances IMCL reduction along with acetyl-CoA metabolism and improves insulin sensitivity in male mice. Endocrinology 159, 3007-3019 (2018).

54. O'Neill, H. M. et al. AMPK phosphorylation of ACC2 is required for skeletal muscle fatty acid oxidation and insulin sensitivity in mice. Diabetologia 57. 1693-1702 (2014).

55. Vijayakumar, A. et al. Absence of carbohydrate response element binding protein in adipocytes causes systemic insulin resistance and impairs glucose transport. Cell Rep. 21, 1021-1035 (2017).

56. Yore, M. M. et al. Discovery of a class of endogenous mammalian lipids with anti-diabetic and antiinflammatory effects. Cell 159, 318-332 (2014).

57. Milstein, S. W. \& Hausberger, F. X. Lipogenesis and carbohydrate utilization; effects of glucose concentration and insulin in rat liver and adipose tissue. Diabetes 5, 89-92 (1956).

58. Richardson, D. K. \& Czech, M. P. Primary role of decreased fatty acid synthesis in insulin resistance of large rat adipocytes. Am. J. Physiol. 234 E182-E189 (1978)

59. Roberts, R. et al. Markers of de novo lipogenesis in adipose tissue: associations with small adipocytes and insulin sensitivity in humans. Diabetologia 52 , 882-890 (2009).

60. Virtanen, K. A. et al. Glucose uptake and perfusion in subcutaneous and visceral adipose tissue during insulin stimulation in nonobese and obese humans. J. Clin. Endocrinol. Metab. 87, 3902-3910 (2002).

61. Czech, M. P. Mechanisms of insulin resistance related to white, beige, and brown adipocytes. Mol. Metab. 34, 27-42 (2020)

62. Herman, M. A et al. A novel ChREBP isoform in adipose tissue regulates systemic glucose metabolism. Nature 484, 333-338 (2012)

63. Fernandez, S. et al. Adipocyte ACLY facilitates dietary carbohydrate handling to maintain metabolic homeostasis in females. Cell Rep. 27, 2772-2784.e6 (2019).

64. Mao, J. et al. aP2-Cre-mediated inactivation of acetyl-CoA carboxylase 1 causes growth retardation and reduced lipid accumulation in adipose tissues. Proc. Natl Acad. Sci. USA 106, 17576-17581 (2009).

65. Brun, T. et al. Evidence for an anaplerotic/malonyl-CoA pathway in pancreatic beta-cell nutrient signaling. Diabetes 45, 190-198 (1996).

66. Cantley, J. et al. Disruption of beta cell acetyl-CoA carboxylase-1 in mice impairs insulin secretion and beta cell mass. Diabetologia 62, 99-111 (2019).

67. Roduit, R. et al. A role for the malonyl-CoA/long-chain acyl-CoA pathway of lipid signaling in the regulation of insulin secretion in response to both fuel and nonfuel stimuli. Diabetes 53, 1007-1019 (2004).

68. Kasper, P. et al. NAFLD and cardiovascular diseases: a clinical review. Clin. Res. Cardiol. 110, 921-937 (2021).

69. Hannou, S. A., Haslam, D. E., McKeown, N. M. \& Herman, M. A. Fructose metabolism and metabolic disease. J. Clin. Invest. 128, 545-555 (2018).

70. Lee, Y. et al. Serial biomarkers of de novo lipogenesis fatty acids and incident heart failure in older adults: the cardiovascular health study. J. Am. Heart Assoc. 9, e014119 (2020).

71. Bouchard-Mercier, A., Rudkowska, I., Lemieux, S., Couture, P. \& Vohl, M. C. Polymorphisms, de novo lipogenesis, and plasma triglyceride response following fish oil supplementation. J. Lipid Res. 54 2866-2873 (2013)

72. Calle, R. A. et al. ACC inhibitor alone or co-administered with a DGAT2 inhibitor in patients with non-aclhoholic fatty liver disease: two parallel, 
placebo-controlled, randomized phase $2 \mathrm{a}$ trial. Nat. Med. 27, 1836-1848 (2021)

First study in humans showing that ACC inhibition lowers HBA1 $c$ and that hypertriglyceridaemia can be avoided by co-administration with a DGAT2 inhibitor.

73. Morieri, M. L. PPARA polymorphism influences the cardiovascular benefit of fenofibrate in type 2 diabetes: findings from ACCORD-lipid. Diabetes 69 771-783 (2020)

74. Baardman, J. et al. Macrophage ATP citrate lyase deficiency stabilizes atherosclerotic plaques. Nat. Commun. 11, 6296 (2020).

75. De Silva, G. S. et al. Circulating serum fatty acid synthase is elevated in patients with diabetes and carotid artery stenosis and is LDL-associated. Atherosclerosis 287, 38-45 (2019).

76. Schneider, J. G. et al. Macrophage fatty-acid synthase deficiency decreases diet-induced atherosclerosis. J. Biol. Chem. 285, 23398-23409 (2010).

77. Sabine, J. R., Abraham, S. \& Chaikoff, I. L. Control of lipid metabolism in hepatomas: insensitivity of rate of fatty acid and cholesterol synthesis by mouse hepatoma BW7756 to fasting and to feedback control. Cancer Res. 27, 793-799 (1967)

78. Freed-Pastor, W. A. et al. Mutant p53 disrupts mammary tissue architecture via the mevalonate pathway. Cell 148, 244-258 (2012).

79. Van de Sande, T., De Schrijver, E., Heyns, W., Verhoeven, G. \& Swinnen, J. V. Role of the phosphatidylinositol 3'-kinase/PTEN/Akt kinase pathway in the overexpression of fatty acid synthase in LNCaP prostate cancer cells. Cancer Res. 62 642-646 (2002)

80. Swinnen, J. V. et al. Stimulation of tumor-associated fatty acid synthase expression by growth factor activation of the sterol regulatory element-binding protein pathway. Oncogene 19, 5173-5181 (2000).

81. Kumar-Sinha, C., Ignatoski, K. W., Lippman, M. E., Ethier, S. P. \& Chinnaiyan, A. M. Transcriptome analysis of HER2 reveals a molecular connection to fatty acid synthesis. Cancer Res. 63, 132-139 (2003).

82. Chang, Y., Wang, J., Lu, X., Thewke, D. P. \& Mason, R. J. KGF induces lipogenic genes through a PI3K and JNK/SREBP-1 pathway in H292 cells. J. Lipid Res. 46 2624-2635 (2005)

83. Yang, Y. A., Han, W. F., Morin, P. J., Chrest, F. J. ¿ Pizer, E. S. Activation of fatty acid synthesis during neoplastic transformation: role of mitogen-activated protein kinase and phosphatidylinositol 3-kinase. Exp. Cell Res. 279, 80-90 (2002).

84. Hawley, S. A. et al. Phosphorylation by Akt within the ST loop of AMPK- 1 down-regulates its activation in tumour cells. Biochem. J. 459, 275-287 (1998).

85. Shackelford, D. B. \& Shaw, R. J. The LKB1-AMPK pathway: metabolism and growth control in tumour suppression. Nat. Rev. Cancer 9, 563-575 (2009).

86. Hatzivassiliou, G. et al. ATP citrate lyase inhibition can suppress tumor cell growth. Cancer Cell 8, 311-321 (2005).

\section{First paper showing that genetic or}

pharmacological inhibition of ACLY with SB-204990 reduces cancer cell proliferation in vitro and in mouse xenografts.

87. $\mathrm{Gu}, \mathrm{L}$. et al. The IKK $\beta$-USP30-ACLY axis controls lipogenesis and tumorigenesis. Hepatology 73 160-174 (2021)

88. Chajes, V., Cambot, M., Moreau, K., Lenoir, G. M. $\&$ Joulin, V. Acetyl-CoA carboxylase alpha is essential to breast cancer cell survival. Cancer Res. 66 5287-5294 (2006).

89. Notarnicola, M. et al. Fatty acid synthase hyperactivation in human colorectal cancer: relationship with tumor side and sex. Oncology 71 , 327-332 (2006)

90. De Piano, M. et al. Lipogenic signalling modulates prostate cancer cell adhesion and migration via modification of Rho GTPases. Oncogene 39, 3666-3679 (2020)

91. Icard, P. et al. ATP citrate lyase: a central metabolic enzyme in cancer. Cancer Lett. 471, 125-134 (2020).

92. Fhu, C. W. \& Ali, A. Fatty acid synthase: an emerging target in cancer. Molecules 25, 3935 (2020).

93. Rae, C., Haberkorn, U., Babich, J. W. \& Mairs, R. J. Inhibition of fatty acid synthase sensitizes prostate cancer cells to radiotherapy. Radiat. Res. 184 , 482-493 (2015)

94. Shah, S. et al. Targeting ACLY sensitizes castrationresistant prostate cancer cells to AR antagonism by impinging on an ACLY-AMPK-AR feedback mechanism. Oncotarget 7, 43713-43730 (2016).
95. Zheng, Y. et al. ATP citrate lyase inhibitor triggers endoplasmic reticulum stress to induce hepatocellular carcinoma cell apoptosis via p-elF2 $\alpha /$ ATF4/CHOP axis J. Cell Mol. Med. 25, 1468-1479 (2021)

96. Qian, X., Hu, J., Zhao, J. \& Chen, H. ATP citrate lyase expression is associated with advanced stage and prognosis in gastric adenocarcinoma. Int. J. Clin. Exp. Med. 8, 7855-7860 (2015).

97. Pearce, E. L., Poffenberger, M. C., Chang, C. H. $\&$ Jones, R. G. Fueling immunity: insights into metabolism and lymphocyte function. Science 342 1242454 (2013)

98. Berod, L. et al. De novo fatty acid synthesis controls the fate between regulatory T and T helper 17 cells Nat. Med. 20, 1327-1333 (2014).

99. Kidani, Y. et al. Sterol regulatory element-binding proteins are essential for the metabolic programming of effector T cells and adaptive immunity. Nat. Immunol. 14, 489-499 (2013)

100. Batista-Gonzalez, A., Vidal, R., Criollo, A. \& Carreño, L. J. New insights on the role of lipid metabolism in the metabolic reprogramming of macrophages. Front. Immunol. 10, 2993 (2020).

101. Lefere, S. \& Tacke, F. Macrophages in obesity and non alcoholic fatty liver disease: crosstalk with metabolism. JHEP Rep. 1, 30-43 (2019).

102. Lauterbach, M. A. et al. Toll-like receptor signaling rewires macrophage metabolism and promotes histone acetylation via ATP-citrate lyase. Immunity $\mathbf{5 1}$ 997-1011.e7 (2019).

103. Covarrubias, A. J. et al. Akt-mTORC1 signaling regulates Acly to integrate metabolic input to control of macrophage activation. eLife 5, e11612 (2016).

104. Osinalde, N. et al. Nuclear phosphoproteomic screen uncovers ACLY as mediator of IL-2-induced proliferation of CD4+T lymphocytes. Mol. Cell Proteom. 15, 2076-2092 (2016).

105. Mamareli, P. et al. Targeting cellular fatty acid synthesis limits T helper and innate lymphoid cell function during intestinal inflammation and infection. Mucosal Immunol. 14, 164-176 (2020).

106. Lee, J. et al. Regulator of fatty acid metabolism, acetyl coenzyme a carboxylase 1 , controls T cell immunity. J. Immunol. 192, 3190-3199 (2014).

107. Yuan, S. et al. SREBP-dependent lipidomic reprogramming as a broad-spectrum antiviral target. Nat. Commun. 10, 120 (2019).

108. van Praag, H. et al. Functional neurogenesis in the adult hippocampus. Nature 415, 1030-1034 (2002)

109. Dimas, P. et al. CNS myelination and remyelination depend on fatty acid synthesis by oligodendrocytes. elife 8, e44702 (2019).

110. Grassi, D. et al. Identification of a highly neurotoxic $\alpha$-synuclein species inducing mitochondrial damage and mitophagy in Parkinson's disease. Proc. Natl Acad. Sci. USA 115, E2634-E2643 (2018).

111. Roncal, J. et al. Adult hippocampal neurogenesis is abundant in neurologically healthy subjects and drops sharply in patients with Alzheimer's disease. Nat. Med. 25, 554-560 (2019).

112. Najmabadi, H. et al. Deep sequencing reveals 50 novel genes for recessive cognitive disorders. Nature 478, 57-63 (2011).

113. Bowers, M. et al. FASN-dependent lipid metabolism links neurogenic stem/progenitor cell activity to learning and memory deficits. Cell Stem Cell 27 98-109.e11 (2020).

114. Ziegler, A. B. et al. Cell-autonomous control of neuronal dendrite expansion via the fatty acid synthesis regulator SREBP. Cell Rep. 21, 3346-3353 (2017).

115. Harayama, T. \& Riezman, H. Understanding the diversity of membrane lipid composition. Nat. Rev. Mol. Cell Biol. 19, 281-296 (2018).

116. Montani, L. et al. De novo fatty acid synthesis by Schwann cells is essential for peripheral nervous system myelination. J. Cell Biol. 217, 1353-1368 (2018).

117. Klingenberg, M. Kinetic study of the tricarboxylate carrier in rat liver mitochondria. Eur. J. Biochem. 26, 587-594 (1972)

118. Halperin, M. L., Robinson, B. H. \& Fritz, I. B. Effects of palmitoyl $\mathrm{COA}$ on citrate and malate transport by rat liver mitochondria. Proc. Natl Acad. Sci. USA 69, 1003-1007 (1972)

119. Palmieri, E. M. et al. Acetylation of human mitochondrial citrate carrier modulates mitochondrial citrate/malate exchange activity to sustain NADPH production during macrophage activation. Biochim. Biophys. Acta 1847, 729-738 (2015).

120. Ma, C. et al. Identification of the substrate binding sites within the yeast mitochondrial citrate transport protein. J. Biol. Chem. 282, 17210-17220

(2007).

Provides the first function-based identification of calcium binding sites within CIC that are crucial for the binding mechanism of $\mathrm{CIC}$ inhibitors.

121. Aluvila, S. et al. The yeast mitochondrial citrate transport protein: molecular determinants of its substrate specificity. J. Biol. Chem. 285 , 27314-27326 (2010)

122. Aluvila, S., Sun, J., Harrison, D. H., Walters, D. E. \& Kaplan, R. S. Inhibitors of the mitochondrial citrate transport protein: validation of the role of substrate binding residues and discovery of the first purely competitive inhibitor. Mol. Pharmacol. 77, 26-34 (2010).

123. Fernandez, H. R. et al. The mitochondrial citrate carrier, SLC25A1, drives stemness and therapy resistance in non-small cell lung cancer. Cell Death Differ. 25, 1239-1258 (2018)

124. Wei, X., Scultz, K., Bazilevsky, G. A., Vogt, A. \& Marmorstein, R. Molecular basis for acetyl-CoA production by ATP-citrate lyase. Nat. Struct. Mol. Biol. 27, 33-41 (2020).

125. Martin, D. B. \& Vagelos, P. R. The mechanism of tricarboxylic acid cycle regulation of fatty acid synthesis. J. Biol. Chem. 237, 1787-1792 (1962).

126. Sun, Q. et al. Regulation on citrate influx and metabolism through inhibiting SLC13A5 and ACLY: a novel mechanism mediating the therapeutic effects of curcumin on NAFLD. J. Agric. Food Chem. 69, 8714-8725 (2021).

127. Joseph, J. W. et al. The mitochondrial citrate/isocitrate carrier plays a regulatory role in glucose-stimulated insulin secretion. J. Biol. Chem. 281, 35624-35632 (2006).

128. Infantino, V., lacobazzi, V., Menga, A. Avantaggiati, M. L. \& Palmieri, F. A key role of the mitochondrial citrate carrier (SLC25A1) in TNF $\alpha$ - and IFN $\gamma$-triggered inflammation. Biochim. Biophys. Acta 1839, 1217-1225 (2014).

129. Assmann, N. et al. SREBP-controlled glucose metabolism is essential for NK cell functional responses. Nat. Immunol. 18, 1197-1206 (2017).

130. Mosaoa, R., Kasprzyk-Pawelec, A., Fernandez, H. R. \& Avantaggiati, M. L. The mitochondrial citrate carrier $\mathrm{SLC} 25 \mathrm{~A} 1 / \mathrm{CIC}$ and the fundamental role of citrate in cancer, inflammation and beyond. Biomolecules 11 , 141 (2021).

131. Jiang, L. et al. Reductive carboxylation supports redox homeostasis during anchorage-independent growth. Nature 532, 255-258 (2016)

132. Catalina-Rodriguez, O. et al. The mitochondrial citrate transporter, $\mathrm{CIC}$, is essential for mitochondrial homeostasis. Oncotarget 3, 1220-1235 (2012).

133. Poolsri, W. A. et al. Combination of mitochondrial and plasma membrane citrate transporter inhibitors inhibits de novo lipogenesis pathway and triggers apoptosis in hepatocellular carcinoma cells. Biomed. Res. Int. 2018, 3683026 (2018).

134. Potapova, I. A., El-Maghrabi, M. R., Doronin, S. V. \& Benjamin, W. B. Phosphorylation of recombinant human ATP:citrate lyase by cAMP-dependent protein kinase abolishes homotropic allosteric regulation of the enzyme by citrate and increases the enzyme activity. Allosteric activation of ATP:citrate lyase by phosphorylated sugars. Biochemistry 39, 1169-1179 (2000).

135. Alexander, M. C., Kowaloff, E. M., Witters, L. A. Dennihy, D. T. \& Avruch, J. Purification of a hepatic 123,000-dalton hormone-stimulated 32P-peptide and its identification as ATP-citrate lyase. J. Biol. Chem. 254, 8052-8056 (1979)

First identification that a peptide phosphorylated by insulin and glucagon in the liver is the citrate cleavage enzyme ACLY and is the same peptide also found in white adipocytes.

136. Pierce, M. W., Palmer, J. L., Keutmann, H. T. $\delta$ Avruch, J. ATP-citrate lyase. Structure of a tryptic peptide containing the phosphorylation site directed by glucagon and the cAMP-dependent protein kinase. J. Biol. Chem. 256, 8867-8870 (1981).

137. Ramakrishna, S., D'Angelo, G. \& Benjamin, W. B. Sequence of sites on ATP-citrate lyase and phosphatase inhibitor 2 phosphorylated by multifunctional protein kinase (a glycogen synthase kinase 3 like kinase). Biochemistry 29, 7617-7624 (1990).

138. Pierce, M. W., Palmer, J. L., Keutmann, H. T., Hall, T. A. \& Avruch, J. The insulin-directed phosphorylation site on ATP-citrate lyase is identical with the site phosphorylated by the cAMP-dependent protein kinase in vitro. J. Biol. Chem. 257, 10681-10686 (1982). 
139. Berwick, D. C., Hers, I., Heesom, K. J., Moule, S. K. \& Tavare, J. M. The identification of ATP-citrate lyase as a protein kinase B (Akt) substrate in primary adipocytes. J. Biol. Chem. 277, 33895-33900 (2002).

140. Martinez Calejman, C. et al. mTORC2-AKT signaling to ATP-citrate lyase drives brown adipogenesis and de novo lipogenesis. Nat. Commun. 11, 575 (2020).

141. White, P. J. et al. The BCKDH kinase and phosphatase integrate BCAA and lipid metabolism via regulation of ATP-citrate lyase. Cell Metab. 27, 1281-1293.e7 (2018).

142. Williams, S. P., Sykes, B. D. \& Bridger, W. A Phosphorus-31 nuclear magnetic resonance study of the active site phosphohistidine and regulatory phosphoserine residues of rat liver ATP-citrate lyase. Biochemistry 24, 5527-5531 (1985)

143. Fan, F. et al. On the catalytic mechanism of human ATP citrate lyase. Biochemistry 51, 5198-5211 (2012).

144. Kumari, R., Deshmukh, R. S. \& Das, S. Caspase-10 inhibits ATP-citrate lyase-mediated metabolic and epigenetic reprogramming to suppress tumorigenesis. Nat. Commun. 10, 4255 (2019).

145. Verschueren, K. H. G. et al. Structure of ATP citrate lyase and the origin of citrate synthase in the Krebs cycle. Nature 568, 571-575 (2019).

Reports high-resolution crystal structures of human ACLY and provides insight into the conformational plasticity of ACLY that is important for regulating catalytic activity.

146. Fatland, B. L. et al. Molecular characterization of a heteromeric ATP-citrate lyase that generates cytosolic acetyl-coenzyme A in Arabidopsis. Plant Physiol. 130 740-756 (2002)

147. Watson, J. A., Fang, M. \& Lowenstein, J. M Tricarballylate and hydroxycitrate: substrate and inhibitor of ATP: citrate oxaloacetate lyase. Arch. Biochem. Biophys. 35, 209-217 (1969).

148. Watson, J. A. \& Lowenstein, J. M. Citrate and the conversion of carbohydrate into fat. J. Biol. Chem 245, 5993-6002 (1970)

149. Triscari, J. \& Sullivan, A. C. Comparative effects of $(-)$-hydroxycitrate and (+)-allo-hydroxycitrate on acetyl CoA carboxylase and fatty acid and cholesterol synthesis in vivo. Lipids 12, 357-363 (1977).

150. Dolle, R. E. et al. Synthesis of novel thiol-containing citric acid analogues. Kinetic evaluation of these and other potential active-site-directed and mechanismbased inhibitors of ATP citrate lyase. J. Med. Chem. 38, 537-543 (1995)

151. Chan, G. W. et al. Purpurone, an inhibitor of ATP-citrate lyase: a novel alkaloid from the marine sponge lotrochota sp. J. Org. Chem. 58, 2544-2546 (1993).

152. Oleynek, J. J. et al. Anthrones, naturally occurring competitive inhibitors of adenosine-triphosphatecitrate lyase. Drug Dev. Res. 36, 35-42 (1995).

153. Ki, S. W. et al. Radicicol binds and inhibits mammalian ATP citrate lyase. J. Biol. Chem. 275, 39231-39236 (2000).

154. Gao, Y., Islam, M. S., Tian, J., Lui, V. W. \& Xiao, D. Inactivation of ATP citrate lyase by cucurbitacin B. a bioactive compound from cucumber inhibits prostate cancer growth. Cancer Lett. 349, 15-25 (2014).

155. Koerner, S. K. Design and synthesis of emodin derivatives as novel inhibitors of ATP-citrate lyase. Eur. J. Med. Chem. 126, 920-928 (2017).

156. Kim, Y. J., Lee, S. A., Myung, S. C., Kim, W. \& Lee, C. S. Radicicol, an inhibitor of Hsp90, enhances TRAILinduced apoptosis in human epithelial ovarian carcinoma cells by promoting activation of apoptosisrelated proteins. Mol. Cell Biochem. 359, 33-43 (2012).

157. Gribble, A. D. et al. ATP-citrate lyase as a target for hypolipidemic intervention. Design and synthesis of 2-substituted butanedioic acids as novel, potent inhibitors of the enzyme. J. Med. Chem. 39 , 3569-3584 (1996).

158. Pearce, N. J. et al. The role of ATP citrate-lyase in the metabolic regulation of plasma lipids. Hypolipidaemic effects of SB-204990, a lactone prodrug of the potent ATP citrate-lyase inhibitor SB-201076. Biochem. J. 334, 113-119 (1998)

159. Jernigan, F. E., Hanai, J. I., Sukhatme, V. P. \& Sun, L. Discovery of furan carboxylate derivatives as novel inhibitors of ATP-citrate lyase via virtual highthroughput screening. Bioorg. Med. Chem. Lett. 27 929-935 (2017)

160. Li, J. J. et al. 2-Hydroxy- $N$-arylbenzenesulfonamides as ATP-citrate lyase inhibitors. Bioorg. Med. Chem. Lett. 17, 3208-3211 (2007).
161. Wei, J. et al. An allosteric mechanism for potent inhibition of human ATP-citrate lyase. Nature $\mathbf{5 6 8}$, 566-570 (2019)

This study demonstrates that NDI-091143 binds next to the ACLY citrate binding site resulting in an extensive conformational change that prevents citrate binding and allosteric activation.

162. Bar-Tana, J., Rose-Kahn, G. \& Srebnik, M. Inhibition of lipid synthesis by beta beta'-tetramethyl-substituted, C14-C22, alpha, omega-dicarboxylic acids in the rat in vivo. J. Biol. Chem. 260, 8404-8410 (1985). First study showing that the dicarboxylic acid MEDICA 16 inhibits both cholesterol and fatty acid synthesis.

163. Rose-Kahn, G. \& Bar-Tana, J. Inhibition of lipid synthesis by beta beta'-tetramethyl-substituted, C14-C22, alpha, omega-dicarboxylic acids in cultured rat hepatocytes. J. Biol. Chem. 260, 8411-8415 (1985).

164. Atkinson, L. L., Kelly, S. E., Russell, J. C., Bar-Tana, J. \& Lopaschuk, G. D. MEDICA 16 inhibits hepatic acetyl-CoA carboxylase and reduces plasma triacylglycerol levels in insulin-resistant JCR: LA-cp rats. Diabetes 51, 1548-1555 (2002).

165. Shurbaji, A. et al. Effect of 3-thiadicarboxylic acid on lipid metabolism in experimental nephrosis. Arterioscler. Thromb. 13, 1580-1586 (1993).

166. Cramer, C. T. et al. Effects of a novel dual lipid synthesis inhibitor and its potential utility in treating dyslipidemia and metabolic syndrome. J. Lipid Res. 45, 1289-1301 (2004)

167. Pinkosky, S. L. et al. Liver-specific ATP-citrate lyase inhibition by bempedoic acid decreases LDL-C and attenuates atherosclerosis. Nat. Commun. 7, 13457 (2016).

Demonstrates that conversion of bempedoic acid into bempedoyl-CoA by a long-chain acyl-CoA synthetase (ACSVL1) expressed in the liver is necessary for suppressing ACLY activity, LDL-cholesterol and atherosclerosis in mice.

168. Sullivan, C. \& Triscari, J. Metabolic regulation as a control for lipid disorders. I. Influence of (-)-hydroxycitrate on experimentally induced obesity in the rodent. Am. J. Clin. Nutr. 30, 767-776 (1977).

169. Asghar, M. et al. Super CitriMax (HCA-SX) attenuates increases in oxidative stress, inflammation, insulin resistance, and body weight in developing obese Zucker rats. Mol. Cell Biochem. 304, 93-99 (2007)

170. Preuss, H. G. et al. Effects of a natural extract of (-)-hydroxycitric acid (HCA-SX) and a combination of HCA-SX plus niacin-bound chromium and Gymnema sylvestre extract on weight loss. Diabetes Obes. Metab. 6, 171-180 (2004).

171. Heymsfield, S. B. et al. Garcinia cambogia (hydroxycitric acid) as a potential antiobesity agent: a randomized controlled trial. JAMA 280, 1596-1600 (1998).

172. Pinkosky, S. L. et al. AMP-activated protein kinase and ATP-citrate lyase are two distinct molecular targets for ETC-1002, a novel small molecule regulato of lipid and carbohydrate metabolism. J. Lipid Res. 54, 134-151 (2013)

173. Banach, M. et al. Association of bempedoic acid administration with atherogenic lipid levels in phase 3 randomized clinical trials of patients with hypercholesterolemia. JAMA Cardiol. 5, 1-12 (2020).

174. Mayorek, N., Kalderon, B., Itach, E. \& Bar-Tana, J. Sensitization to insulin induced by beta,beta'-methylsubstituted hexadecanedioic acid (MEDICA 16) in obese Zucker rats in vivo. Diabetes 46, 1958-1964 (1997).

175. Russell, J. C. et al. Development of insulin resistance in the JCR:LA-cp rat: role of triacylglycerols and effects of MEDICA 16. Diabetes 47, 770-778 (1998).

176. Masson, W., Lobo, M., Lavalle-Cobo, A., Masson, G. \& Molinero, G. Effect of bempedoic acid on new onset or worsening diabetes: a meta-analysis. Diabetes Res. Clin. Pract. 168, 108369 (2020).

177. Russell, J. C. et al. Hypolipidemic effect of beta beta'-tetramethyl hexadecanedioic acid (MEDICA 16) in hyperlipidemic JCR:LA-corpulent rats. Arterioscler. Thromb. 11, 602-609 (1991).

178. Russell, J. C. et al. Inhibition of atherosclerosis and myocardial lesions in the JCR:LA-cp rat by beta, beta'-tetramethylhexadecanedioic acid (MEDICA 16). Arterioscler. Thromb. Vasc. Biol. 15, 918-923 (1995).

179. Burke, A. C. et al. Bempedoic acid lowers low-density lipoprotein cholesterol and attenuates atherosclerosis in low-density lipoprotein receptor-deficient $\left(L D L R^{+/}\right.$ and $L D L R^{-1}$ ) Yucatan miniature pigs. Arterioscler. Thromb. Vasc. Biol. 38, 1178-1190 (2018).
180. Pinkosky, S. L., Groot, P. H. E., Lalwani, N. D. \& Steinberg, G. R. Targeting ATP-citrate lyase in hyperlipidemia and metabolic disorders. Trends $\mathrm{Mol}$. Med. 23, 1047-1063 (2017)

181. Ray, K. K. et al. Safety and efficacy of bempedoic acid to reduce LDL cholesterol. N. Engl. J. Med. 380 , 1022-1032 (2019).

182. Laufs, U. et al. Efficacy and safety of bempedoic acid in patients with hypercholesterolemia and statin intolerance. J. Am. Heart Assoc. 8, e011662 (2019). Together with Ray et al. (2019), provides evidence that bempedoic acid safely lowers LDL-cholestero in patients on maximally tolerated statin therapy or intolerant to statins.

183. Abolhassani, M. et al. Screening of well-established drugs targeting cancer metabolism: reproducibility of the efficacy of a highly effective drug combination in mice. Invest. N. Drugs 30, 1331-1342 (2012).

184. Tong, L. Acetyl-coenzyme A carboxylase: crucial metabolic enzyme and attractive target for drug discovery. Cell Mol. Life Sci. 62, 1784-1803 (2005)

185. Halestrap, A. P. \& Denton, R. M. Hormonal regulation of adipose-tissue acetyl-Coenzyme A carboxylase by changes in the polymeric state of the enzyme. The role of long-chain fatty acyl-coenzyme A thioesters and citrate. Biochem. J. 142, 365-377 (1974).

186. Carlson, C. A. \& Kim, K. H. Regulation of hepatic acetyl coenzyme A carboxylase by phosphorylation and dephosphorylation. J. Biol. Chem. 248, 378-380 (1973).

First study demonstrating that phosphorylation of ACC inhibits enzyme activity and could override allosteric activation by citrate.

187. Lent, B. A., Lee, K. H. \& Kim, K. H. Regulation of rat liver acetyl-CoA carboxylase. Stimulation of phosphorylation and subsequent inactivation of liver acetyl-CoA carboxylase by cyclic 3':5'-monophosphate and effect on the structure of the enzyme. J. Biol. Chem. 253, 8149-8156 (1978).

188. Munday, M. R., Campbell, D. G., Carling, D. \& Hardie, D. G. Identification by amino acid sequencing of three major regulatory phosphorylation sites on rat acetyl-CoA carboxylase. Eur. J. Biochem. 175, 331-338 (1988)

189. Lally, J. S. V. et al. Inhibition of acetyl-coa carboxylase by phosphorylation or the inhibitor ND-654 suppresses lipogenesis and hepatocellular carcinoma. Cell Metab. 29, 174-182.e5 (2019).

190. Pinkosky, S. L. et al. Long-chain fatty acyl-CoA esters regulate metabolism via allosteric control of AMPK $\beta$ isoforms. Nat. Metab. 2, 873-881 (2020).

Demonstrates that fatty acyl-CoAs allosterically activate AMPK and that subsequent phosphorylation of ACC is required to increase fatty acid oxidation in mice.

191. Wei, J. \& Tong, L. Crystal structure of the 500-kDa yeast acetyl-CoA carboxylase holoenzyme dimer. Nature 526, 723-727 (2015)

192. Zhang, H., Yang, Z., Shen, Y. \& Tong, L. Crystal structure of the carboxyltransferase domain of acetylcoenzyme A carboxylase. Science 299, 2064-2067 (2003).

193. Hunkele, M. et al. Structural basis for regulation of human acetyl-CoA carboxylase. Nature $\mathbf{5 5 8}$ 470-474 (2018)

Reports novel insights detailing dynamic interactions that occur in human ACC upon exposure to allosteric regulators such as citrate and palmityol-CoA

194. Wei, J. et al. A unified molecular mechanism for the regulation of acetyl-CoA carboxylase by phosphorylation. Cell Discov. 2, 16044 (2016).

195. Vahlensieck, H. F., Pridzun, L., Reichenbach, H. \& Hinnen, A. Identification of the yeast ACC1 gene product (acetyl-CoA carboxylase) as the target of the polyketide fungicide soraphen A. Curr. Genet. 25 95-100 (1994).

196. Shen, Y., Volrath, S. L., Weatherly, S. C., Elich, T. D. $\&$ Tong, L. A mechanism for the potent inhibition of eukaryotic acetyl-coenzyme A carboxylase by soraphen A, a macrocyclic polyketide natural product. Mol. Cell 16, 881-891 (2004).

197. Bianchi, A., Evans, J. L., Nordlund, A. C., Watts, T. D. $\&$ Witters, L. A. Acetyl-CoA carboxylase in Reuber hepatoma cells: variation in enzyme activity, insulin regulation, and cellular lipid content. J. Cell Biochem. 48, 86-97 (1992).

198. Harwood, H. J. Jr et al. Isozyme-nonselective $\mathrm{N}$-substituted bipiperidylcarboxamide acetyl-CoA carboxylase inhibitors reduce tissue malonyl-CoA concentrations, inhibit fatty acid synthesis, and increase fatty acid oxidation in cultured cells 
and in experimental animals. J. Biol. Chem 278 37099-37111 (2003).

First study describing the discovery of a synthetic isozyme-nonselective ACC inhibitor, CP-610431, and its analogue CP-640186, by high-throughput inhibition screening and that this compound reduced fatty acid synthesis and increased fatty acid oxidation

199. Zhang, H., Tweel, B., Li, J. \& Tong, L. Crystal structure of the carboxyltransferase domain of acetyl-coenzyme A carboxylase in complex with CP-640186. Structure 12, 1683-1691 (2004)

200. Chonan, T. et al. (4-Piperidinyl)-piperazine: a new platform for acetyl-CoA carboxylase inhibitors. Bioorg. Med. Chem. Lett. 19, 6645-6648 (2009).

201. Yamashita, T. et al. Design, synthesis, and structureactivity relationships of spirolactones bearing 2-ureidobenzothiophene as acetyl-CoA carboxylases inhibitors. Bioorg. Med. Chem. Lett. 21, 6314-6318 (2011)

202. Kamata, M. et al. Design, synthesis, and structure activity relationships of novel spiro-piperidines as acetyl-CoA carboxylase inhibitors. Bioorg. Med. Chem. Lett. 22, 3643-3647 (2012).

203. Kamata, M. et al. Symmetrical approach of spiropyrazolidinediones as acetyl-CoA carboxylase inhibitors. Bioorg. Med. Chem. Lett. 22, 4769-4772 (2012).

204. Gao, Y. S. et al. WZ66, a novel acetyl-CoA carboxylase inhibitor, alleviates nonalcoholic steatohepatitis (NASH) in mice. Acta Pharmacol. Sin. 41, 336-347 (2020).

205. Bergman, A. et al. Safety, tolerability, pharmacokinetics, and pharmacodynamics of a liver-targeting acetyl-CoA carboxylase inhibitor (PF-05221304): a three-part randomized phase 1 study. Clin. Pharmacol. Drug Dev. 9, 514-526 (2020)

206. Harriman, G. et al. Acetyl-CoA carboxylase inhibition by ND-630 reduces hepatic steatosis, improves insulin sensitivity, and modulates dyslipidemia in rats. Proc. Natl Acad. Sci. USA 113, E1796-E1805 (2016).

Describes the development of a new ACC inhibitor (GS-0976 (Firsocostat)) that inhibits dimerization by binding to and mimicking the AMPK phosphorylation site. This compound inhibited DNL, increased fatty acid oxidation and improved insulin sensitivity in rodents. In subsequent studies, related molecules were shown to reduce non-smallcell lung cancer (Svensson et al. (2006)) and hepatocellular carcinoma (Lally et al. (2019).

207. Svensson, R. U. et al. Inhibition of acetyl-CoA carboxylase suppresses fatty acid synthesis and tumor growth of non-small-cell lung cancer in preclinical models. Nat. Med. 22, 1108-1119 (2016)

208. Mizojiri, R. et al. Discovery of novel selective acetylCoA carboxylase (ACC) 1 inhibitors. J. Med. Chem. 61 , 1098-1117 (2018)

209. Mizojiri, R. et al. Design and synthesis of a novel $1 \mathrm{H}$-pyrrolo[3,2-b]pyridine-3-carboxamide derivative as an orally available ACC1 inhibitor. Bioorg. Med. Chem. 27, 2521-2530 (2019).

210. Gu, Y. G. et al. Synthesis and structure-activity relationships of $\mathrm{N}-\{3-[2-(4-a)$ koxyphenoxy)thiazol5-yl]-1- methylprop-2-ynyl $\}$ carboxy derivatives as selective acetyl-CoA carboxylase 2 inhibitors. J. Med. Chem. 49, 3770-3773 (2006).

211. Gu, Y. G. et al. N-\{3-[2-(4-alkoxyphenoxy)thiazol-5-yl]1-methylprop-2-ynyl $\}$ carboxy derivatives as acetyl-coA carboxylase inhibitors-improvement of cardiovascular and neurological liabilities via structural modifications. J. Med. Chem. $\mathbf{5 0}$ 1078-1082 (2007)

212. Glund, S. et al. Inhibition of acetyl-CoA carboxylase 2 enhances skeletal muscle fatty acid oxidation and improves whole-body glucose homeostasis in $\mathrm{db} / \mathrm{db}$ mice. Diabetologia 55, 2044-2053 (2012).

213. Nishiura, Y. et al. Discovery of a novel olefin derivative as a highly potent and selective acetyl-CoA carboxylase 2 inhibitor with in vivo efficacy. Bioorg. Med. Chem. Lett. 28, 2498-2503 (2018)

214. Schreurs, M. et al. Soraphen, an inhibitor of the acetyl-CoA carboxylase system, improves peripheral insulin sensitivity in mice fed a high-fat diet. Diabetes Obes. Metab. 11, 987-991 (2009).

215. Ronnebaum, S. M. et al. Chronic suppression of acetyl-CoA carboxylase 1 in beta-cells impairs insulin secretion via inhibition of glucose rather than lipid metabolism. J. Biol. Chem. 283, 14248-14256 (2008).

216. Liu, T., Gou, L., Yan, S. \& Huang, T. Inhibition of acetyl-CoA carboxylase by PP-7a exerts beneficia effects on metabolic dysregulation in a mouse model of diet-induced obesity. Exp. Ther. Med. 20, 521-529 (2020).

217. Griffith, D. A. et al. Decreasing the rate of metabolic ketone reduction in the discovery of a clinical acetylCoA carboxylase inhibitor for the treatment of diabetes. J. Med. Chem. 57, 10512-10526 (2014).

218. Ross, T. T. et al. Acetyl-CoA carboxylase inhibition improves multiple dimensions of NASH pathogenesis in model systems. Cell Mol. Gastroenterol. Hepatol. 10, 829-851 (2020)

219. Huard, K. et al. Optimizing the benefit/risk of acetyl-CoA carboxylase inhibitors through liver targeting. J. Med. Chem. 63, 10879-10896 (2020).

220. Zhang, J. et al. Molecular profiling reveals a common metabolic signature of tissue fibrosis. Cell Rep. Med. 1, 100056 (2020)

221. Matsumoto, M. et al. Acetyl-CoA carboxylase 1 and 2 inhibition ameliorates steatosis and hepatic fibrosis in a MC4R knockout murine model of nonalcoholic steatohepatitis. PLoS ONE 15, e0228212 (2020).

222. Bates, J. et al. Acetyl-CoA carboxylase inhibition disrupts metabolic reprogramming during hepatic stellate cell activation. J. Hepatol. 73, 896-905 (2020).

223. Stiede, K. et al. Acetyl-coenzyme A carboxylase inhibition reduces de novo lipogenesis in overweight male subjects: a randomized, double-blind, crossover study. Hepatology 66, 324-334 (2017) First study in humans showing that ACC inhibition (with ND-630/GS-0976) reduces liver DNL.

224. Loomba, R et al. GS-0976 reduces hepatic steatosis and fibrosis markers in patients with nonalcoholic fatty liver disease. Gastroenterology 155, 1463-1473.e6 (2018).

First study in humans showing that ACC inhibition reduces liver fat and markers of fibrosis.

225. Loomba, R. et al. Combination therapies including cilofexor and firsocostat for bridging fibrosis and cirrhosis attributable to NASH. Hepatology 73 625-643 (2021)

\section{Phase II clinical trial demonstrating that a} combination of ND-630 and a FXR agonist cilofexor reduces liver fibrosis in people with NASH.

226. Waring, J. F. et al. Gene expression analysis in rats treated with experimental acetyl-coenzyme A carboxylase inhibitors suggests interactions with the peroxisome proliferator-activated receptor alpha pathway. J. Pharmacol. Exp. Ther. 324, 507-516 (2008).

227. Goedeke, L. et al. Acetyl-CoA carboxylase inhibition reverses NAFLD and hepatic insulin resistance but promotes hypertriglyceridemia in rodents. Hepatology 68, 2197-2211 (2018)

228. Corominas-Faja, B. et al. Chemical inhibition of acetyl-CoA carboxylase suppresses self-renewal growth of cancer stem cells. Oncotarget 5 8306-8316 (2014).

229. Li, S. et al. TOFA suppresses ovarian cancer cell growth in vitro and in vivo. Mol. Med. Rep. 8 373-378 (2013).

230. Hess, D., Chisholm, J. W. \& Igal, R. A. Inhibition of stearoylCoA desaturase activity blocks cell cycle progression and induces programmed cell death in lung cancer cells. PLoS ONE 5, e11394 (2010).

231. Martínez-Montañés, F. et al. Phosphoproteomic analysis across the yeast life cycle reveals control of fatty acyl chain length by phosphorylation of the fatty acid synthase complex. Cell Rep. 32, 108024 (2020).

232. Jin, Q. et al. Fatty acid synthase phosphorylation: a novel therapeutic target in HER2-overexpressing breast cancer cells. Breast Cancer Res. 12, R96 (2010).

233. Sun, T., Liu, Z. \& Yang, O. The role of ubiquitination and deubiquitination in cancer metabolism. Mol. Cancer 19, 146 (2020).

234. Smith, S., Witkowski, A. \& Joshi, A. K. Structural and functional organization of the animal fatty acid synthase. Prog. Lipid Res. 42, 289-317 (2003).

235. Hiltunen, J. K. et al. Mitochondrial fatty acid synthesis type II: more than just fatty acids. J. Biol. Chem. 284 , 9011-9015 (2009)

236. White, S. W., Zheng, J., Zhang, Y. M. \& Rock The structural biology of type II fatty acid biosynthesis. Annu. Rev. Biochem. 74, 791-831 (2005).

237. Maier, T., Leibundgut, M. \& Ban, N. The crystal structure of a mammalian fatty acid synthase. Science 321, 1315-1322 (2008) Report of the crystal structure of full mammalian FAS and molecular details of active sites.
238. Omura, S. The antibiotic cerulenin, a novel tool for biochemistry as an inhibitor of fatty acid synthesis. Bacteriol. Rev. 40, 681-697 (1976).

239. Kuhajda, F. P. et al. Synthesis and antitumor activity of an inhibitor of fatty acid synthase. Proc. Natl Acad. Sci. USA 97, 3450-3454 (2000).

First paper describing the generation of the FAS inhibitor $\mathrm{C75}$ and that inhibiting DNL in cancer cells using this compound reduces cell proliferation

240. Cheng, F., Wang, Q., Chen, M., Quiocho, F. A. \& Ma, J. Molecular docking study of the interactions between the thioesterase domain of human fatty acid synthase and its ligands. Proteins 70, 1228-1234 (2008).

241. Kridel, S. J., Axelrod, F., Rozenkrantz, N. \& Smith, J. W. Orlistat is a novel inhibitor of fatty acid synthase with antitumor activity. Cancer Res. 64, 2070-2075 (2004).

242. Hill, T. K. et al. Development of a self-assembled nanoparticle formulation of orlistat, Nano-ORL, with increased cytotoxicity against human tumor cell lines. Mol. Pharm. 13, 720-728 (2016)

243. Bhargava-Shah, A., Foygel, K., Devulapally, R. \& Paulmurugan, R. Orlistat and antisense-miRNA-loaded PLGA-PEG nanoparticles for enhanced triple negative breast cancer therapy. Nanomedicine 11, 235-247 (2016).

244. Paulmurugan, R. et al. Folate receptor-targeted polymeric micellar nanocarriers for delivery of orlistat as a repurposed drug against triple-negative breast cancer. Mol. Cancer Ther. 15, 221-231 (2016).

245. Alwarawrah, Y. et al. Fasnall, a selective FASN inhibitor, shows potent anti-tumor activity in the MMTV-Neu model of HER2(+) breast cancer. Cell Chem. Biol. 23, 678-688 (2016).

246. Singha, P. K. et al. Evaluation of FASN inhibitors by a versatile toolkit reveals differences in pharmacology between human and rodent FASN preparations and in antiproliferative efficacy in vitro vs. in situ in human cancer cells. Eur. J. Pharm. Sci. 149, 105321 2020).

247. Zadra, G. et al. Inhibition of de novo lipogenesis targets androgen receptor signaling in castrationresistant prostate cancer. Proc. Natl Acad. Sci. USA 116, 631-640 (2019)

248. Hardwicke, M. A. et al. A human fatty acid synthase inhibitor binds $\beta$-ketoacyl reductase in the ketosubstrate site. Nat. Chem. Biol. 10, 774-779 (2014).

249. Kley, J. T., Mack, J., Hamilton, B., Scheuerer, S. \& Redemann, N. Discovery of BI 99179 , a potent and selective inhibitor of type I fatty acid synthase with central exposure. Bioorg. Med. Chem. Lett. 21 5924-5927 (2011).

250. Ventura, R. et al. Inhibition of de novo palmitate synthesis by fatty acid synthase induces apoptosis in tumor cells by remodeling cell membranes, inhibiting signaling pathways, and reprogramming gene expression. EBioMedicine 2, 808-824 (2015).

251. Beysen, C. et al. Inhibition of fatty acid synthase with FT-4101 safely reduces hepatic de novo lipogenesis and steatosis in obese subjects with non-alcoholic fatty liver disease: results from two early-phase randomized trials. Diabetes Obes. Metab. 23, 700-710 (2021). First clinical findings that the FAS inhibitor FT-4101 reduces steatosis in people with NAFLD.

252. Loftus, T. M. et al. Reduced food intake and body weight in mice treated with fatty acid synthase inhibitors. Science 288, 2379-2381 (2000). First paper indicating that regulating malony-CoA in the hypothalamus is important for regulating energy intake.

253. Makimura, H. et al. Cerulenin mimics effects of leptin on metabolic rate, food intake, and body weight independent of the melanocortin system, but unlike leptin, cerulenin fails to block neuroendocrine effects of fasting. Diabetes 50, 733-739 (2001).

254. Gao, S. \& Lane, M. D. Effect of the anorectic fatty acid synthase inhibitor $\mathrm{C} 75$ on neuronal activity in the hypothalamus and brainstem. Proc. Natl Acad. Sci. USA 100, 5628-5633 (2003).

255. Thupari, J. N., Landree, L. E., Ronnett, G. V. \& Kuhajda, F. P. C75 increases peripheral energy utilization and fatty acid oxidation in diet-induced obesity. Proc. Natl Acad. Sci. USA 99, 9498-9502 (2002).

256. Thupari, J. N., Kim, E. K., Moran, T. H., Ronnett, G. V. $\Sigma$ Kuhajda, F. P. Chronic C75 treatment of dietinduced obese mice increases fat oxidation and reduces food intake to reduce adipose mass. $A m$. J. Physiol Endocrinol Metab. 287 , E97-E104 (2004).

257. Shimokawa, T., Kumar, M. V. \& Lane, M. D. Effect of a fatty acid synthase inhibitor on food intake and expression of hypothalamic neuropeptides. Proc. Natl Acad. Sci. USA 99, 66-71 (2002). 
258. Syed-Abdul, M. M. et al. Fatty acid synthase inhibitor TVB-2640 reduces hepatic de novo lipogenesis in males with metabolic abnormalities. Hepatology $\mathbf{7 2}$, 103-118 (2020)

First clinical findings indicating that inhibiting FAS in individuals with obesity using TVB-2640 suppresses liver DNL with minimal adverse events.

259. Pandey, P. R., Liu, W., Xing, F., Fukuda, K. \& Watabe, K. Anti-cancer drugs targeting fatty acid synthase (FAS). Recent Pat. Anticancer Drug Discov. 7, 185-197 (2012).

260. Pizer, E. S. et al. Inhibition of fatty acid synthesis delays disease progression in a xenograft model of ovarian cancer. Cancer Res. 56, 1189-1193 (1996).

261. Ho, T. S. et al. Fatty acid synthase inhibitors cerulenin and $\mathrm{C} 75$ retard growth and induce caspase-dependent apoptosis in human melanoma A-375 cells. Biomed. Pharmacother. 61, 578-587 (2007).

262. Elix, C. C. et al. Peroxisome proliferator-activated receptor gamma controls prostate cancer cell growth through AR-dependent and independent mechanisms. Prostate 80, 162-172 (2020).

263. Ferraro, G. B. et al. Fatty acid synthesis is required for breast cancer brain metastasis. Nat. Cancer 2 414-428 (2021).

264. Falchook, G. et al. First-in-human study of the safety, pharmacokinetics, and pharmacodynamics of firstin-class fatty acid synthase inhibitor TVB-2640 alone and with a taxane in advanced tumors. EClinicalMedicine 34, 100797 (2021)

265. Beigneux, A. P. et al. ATP-citrate lyase deficiency in the mouse. J. Biol. Chem. 279, 9557-9564 (2004).

266. Abu-Elheiga, L. et al. Mutant mice lacking acetyl-CoA carboxylase 1 are embryonically lethal. Proc. Natl Acad. Sci. USA 102, 12011-12016 (2005).

267. Chirala, S. S. et al. Fatty acid synthesis is essential in embryonic development: fatty acid synthase null mutants and most of the heterozygotes die in utero. Proc. Natl Acad. Sci. USA 100, 6358-6363 (2003).

268. Palmieri, F., Scarcia, P. \& Monné, M. Diseases caused by mutations in mitochondrial carrier genes SLC25: a review. Biomolecules 10, 655 (2020). Together with Beigneux et al. (2004), Abu-Elheiga et al. (2005) and Chirala et al. (2003), provides evidence demonstrating a crucial role for DNL in normal embryonic development.

269. Esquejo, R. M. et al. Activation of Liver AMPK with PF-06409577 corrects NAFLD and lowers cholesterol in rodent and primate preclinical models. EBioMedicine 31, 122-132 (2018).

270. Gluais-Dagorn, P. et al. Direct AMPK activation corrects NASH in rodents through metabolic effects and direct action on inflammation and fibrogenesis. Hepatol. Commun. 6, 101-119 (2021).

271. Bruning, U. et al. Impairment of angiogenesis by fatty acid synthase Inhibition Involves mTOR malonylation. Cell Metab. 28, 866-880.e15 (2018).

272. Colak, G. et al. Proteomic and biochemical studies of lysine malonylation suggest its malonic aciduriaassociated regulatory role in mitochondrial function and fatty acid oxidation. Mol. Cell Proteom. 14 3056-3071 (2015).

273. Ishiguro, T. et al. Malonylation of histone $\mathrm{H} 2 \mathrm{~A}$ at lysine 119 inhibits Bub 1-dependent $\mathrm{H} 2 \mathrm{~A}$ phosphorylation and chromosomal localization of shugoshin proteins. Sci. Rep. 8, 7671 (2018).

274. Covarrubias, S. et al. Malonylation of GAPDH is an inflammatory signal in macrophages. Nat. Commun. 10, 338 (2019)

275. Wellen, K. E. et al. ATP-citrate lyase links cellular metabolism to histone acetylation. Science $\mathbf{3 2 4}$, 1076-1080 (2009).

The first paper linking nutrient availability with histone acetylation through regulation of ACLY activity.
276. Kamphorst, J. J. et al. Hypoxic and Ras-transformed cells support growth by scavenging unsaturated fatty acids from lysophospholipids. Proc. Natl Acad. Sci. USA 110, 8882-8887 (2013)

277. Watt, M. J. et al. Suppressing fatty acid uptake has therapeutic effects in preclinical models of prostate cancer. Sci. Transl Med. 11, eaau5758 (2019).

278. Zaidi, N., Royaux, I., Swinnen, J. V. \& Smans, K. ATP citrate lyase knockdown induces growth arrest and apoptosis through different cell- and environmentdependent mechanisms. Mol. Cancer Ther. 11. 1925-1935 (2012).

279. Nazy, I., Arnold, D. M. \& Steinberg, G. R. The mega-importance of de novo lipogenesis in platelet production. Nat. Metab. 2, 999-1000 (2020)

280. Eissing, L. et al. De novo lipogenesis in human fat and liver is linked to ChREBP- $\beta$ and metabolic health. Nat. Commun. 4, 1528 (2013).

281. Abdul-Wahed, A., Guilmeau, S. \& Postic, C. Sweet sixteenth for ChREBP: established roles and future goals. Cell Metab. 26, 324-341 (2017).

282. Ye, J. \& DeBose-Boyd, R. A. Regulation of cholesterol and fatty acid synthesis. Cold Spring Harb. Perspect. Biol. 3, a004754 (2011).

283. Chen, G., Liang, G., Ou, J., Goldstein, J. L. \& Brown, M. S. Central role for liver $\mathrm{X}$ receptor in insulin-mediated activation of Srebp-1c transcription and stimulation of fatty acid synthesis in liver. Proc. Natl Acad. Sci. USA 101, 11245-11250 (2004).

284. Cha, J. Y. \& Repa, J. J. The liver X receptor (LXR) and hepatic lipogenesis. The carbohydrate-response element-binding protein is a target gene of LXR. J. Biol. Chem. 282, 743-751 (2007).

285. Denechaud, P. D. et al. ChREBP, but not LXRs, is required for the induction of glucose-regulated genes in mouse liver. J. Clin. Invest. 118, 956-964 (2008).

286. Koutsoudakis, G. et al. Soraphen A: a broad-spectrum antiviral natural product with potent anti-hepatitis $C$ virus activity. J. Hepatol. 63, 813-821 (2015).

287. Fleta-Soriano, E. et al. The myxobacterial metabolite soraphen a inhibits HIV-1 by reducing virus production and altering virion composition. Antimicrob. Agents Chemother. 61, e00739-17 (2017)

288. Merino-Ramos, T. et al. Modification of the host cell lipid metabolism induced by hypolipidemic drugs targeting the acetyl coenzyme a carboxylase impairs west nile virus replication. Antimicrob. Agents Chemother. 60, 307-315 (2015)

289. Gaunt, E. R., Cheung, W., Richards, J. E., Lever, A. \& Desselberger, U. Inhibition of rotavirus replication by downregulation of fatty acid synthesis. J. Gen. Virol. 94, 1310-1317 (2013)

290. Li, Y., Webster-Cyriaque, J., Tomlinson, C. C., Yohe, M $\&$ Kenney, S. Fatty acid synthase expression is induced by the Epstein-Barr virus immediate-early protein BRLF1 and is required for lytic viral gene expression. J. Virol. 78, 4197-4206 (2004).

291. Hitakarun, A. et al. Evaluation of the antiviral activity of orlistat (tetrahydrolipstatin) against dengue virus, Japanese encephalitis virus, zika virus and chikungunya virus. Sci. Rep. 10, 1499 (2020)

292. Tanner, J. E. \& Alfieri, C. The fatty acid lipid metabolism nexus in COVID-19. Viruses 13, 90 (2021).

293. Chu, J. et al. Pharmacological inhibition of fatty acid synthesis blocks SARS-CoV-2 replication. Nat. Metab. 3, 1466-1475 (2021). Indicates that FAS inhibition may be beneficial against COVID-19.

294. Hunt, D. W. et al. Inhibition of sebum production with the acetyl coenzyme a carboxylase inhibitor olumacostat glasaretil. J. Invest. Dermatol. 137, 1415-1423 (2017).
295. Raha, S. et al. Disruption of de novo fatty acid synthesis via acetyl-CoA carboxylase 1 inhibition prevents acute graft-versus-host disease. Eur. J. Immunol. 46, 2233-2238 (2016).

296. Wang, X. et al. ACC1 (Acetyl Coenzyme A Carboxylase 1) is a potential immune modulatory target of cerebral ischemic. Stroke Stroke $\mathbf{5 0}$ 1869-1878 (2019).

297. Gross, A. S. et al. Acetyl-CoA carboxylase 1-dependent lipogenesis promotes autophagy downstream of AMPK. J. Biol. Chem. 294, 12020-12039 (2019).

298. Glatzel, D. K. et al. Acetyl-CoA carboxylase 1 regulates endothelial cell migration by shifting the phospholipid composition. J. Lipid Res. 59, 298-311 (2018).

299. Ibitokou, S. A. et al. Early inhibition of fatty acid synthesis reduces generation of memory precursor effector T cells in chronic infection. J. Immunol. 200, 643-656 (2018).

300. Rymut, S. M. et al. Acetyl-CoA carboxylase inhibition regulates microtubule dynamics and intracellular transport in cystic fibrosis epithelial cells. Am. J. Physiol. Lung Cell Mol. Physiol. 316, L1081-L1093 (2019).

\section{Acknowledgements}

G.R.S. is supported by the Canadian Institutes of Health Research (201709FDN-CEBA-116200 to G.R.S.), Diabetes Canada (DI-5-17-5302-CS), a Tier 1 Canada Research Chair and a J. Bruce Duncan Endowed Chair in Metabolic Diseases. B.B. was supported by a MGDII Health Innovation, Commercialization and Entrepreneurship fellowship. Protein crystal structures were obtained from the RCSB protein databank (rcsb.org) for ACLY (PDB ID 6POF), ACC (PDB ID 5CSK) and FAS (PDB ID 2VZ8) or UniProt (uniprot.org) for CIC (P53007). The authors apologize to researchers whose relevant work could not be cited in the present review owing to space limitations.

\section{Competing interests}

G.R.S. has received research funding from Esperion Therapeutics, Espervita Therapeutics, Poxel Pharmaceuticals and Novo Nordisk, honoraria and/or consulting fees from Astra Zeneca, Eli-Lilly, Esperion Therapeutics, Poxel Pharmaceuticals, Merck and is a founder and shareholder of Espervita Therapeutics. S.L.P. is employed by Esperion Therapeutics and is a shareholder. B.B. declares no competing interests.

\section{Publisher's note}

Springer Nature remains neutral with regard to jurisdictional claims in published maps and institutional affiliations.

\section{Supplementary information}

The online version contains supplementary material available at https://doi.org/10.1038/s41573-021-00367-2.

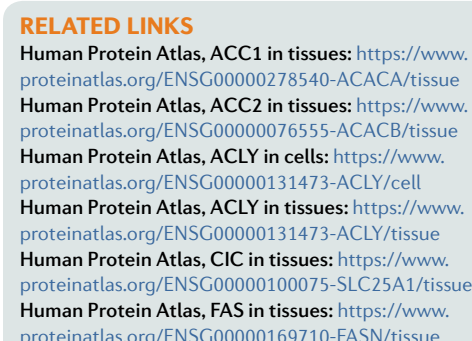

proteinatlas.org/ENSG00000169710-FASN/tissue

(c) Springer Nature Limited 2022 